EPJ manuscript No.

(will be inserted by the editor)

\title{
Invariant Amplitudes for Pion Electroproduction
}

\author{
B. Pasquini ${ }^{1}$, D. Drechsel ${ }^{2}$, L. Tiator ${ }^{2}$ \\ 1 Dipartimento di Fisica Nucleare e Teorica, Universitá degli Studi di Pavia; INFN, Sezione di Pavia, Pavia, Italy \\ ${ }^{2}$ Institut für Kernphysik, Johannes Gutenberg-Universität, D-55099 Mainz
}

$12 / \mathrm{Dec} / 2007$

\begin{abstract}
The invariant amplitudes for pion electroproduction on the nucleon are evaluated by dispersion relations at constant $t$ with MAID as input for the imaginary parts of these amplitudes. In the threshold region these amplitudes are confronted with the predictions of several low-energy theorems derived in the soft-pion limit. In general agreement with Chiral Perturbation Theory, the dispersive approach yields large corrections to these theorems because of the finite pion mass.
\end{abstract}

PACS. 11.55.Fv , 11.55.Hx, 13.40.Gp, 13.60.Le, 14.20.Gk, 25.20.Lj, 25.30.Rw

\section{Introduction}

In two recent publications we have studied pion photoproduction on the nucleon in the framework of fixed- $t$ dispersion relations [1,2]. In particular, we have concentrated on the threshold region in which the results can be compared to both precision data and predictions of baryon chiral perturbation theory (ChPT). The dispersion relations (DRs) are based on a set of 4 photoproduction amplitudes $A_{i}(\nu, t)$ depending on ' energy and momentum transfer described by the Lorentz ' invariant variables $\nu$ and $t$, respectively. These relations are Lorentz and gauge invariant by construction, and unitarity is implemented by constructing the real parts of the amplitudes from the imaginary (absorptive) parts via the dispersion integrals $3,4,5,6$. It is possible to evaluate these amplitudes also outside of the physical region by analytic continuation. In particular, the dispersive amplitudes for sub-threshold kinematics are regular functions in a region of small $\nu$ and $t$ values, and therefore they can be expanded in a power series about the origin of the Mandelstam plane $(\nu=0, t=0)$. Comparing this series with the tree and loop contributions of relativistic baryon ChPT [7,8, 9, 10,11] one can read off the required low-energy constants (LECs) of that field theory, which up to now have been fixed by resonance saturation models or fits to the threshold data. In our present work we use MAID05 12 as input for the absorptive parts of the amplitudes, which are obtained over the full resonance region up to center-of-mass (c.m.) energies of $\mathrm{W}=2.2 \mathrm{GeV}$ by a global fit to the pion photoproduction data. With few exceptions the results compare favorably with the experimental data and the predictions of $\mathrm{ChPT}$ in the threshold region.
Another interesting aspect is the comparison with sum rules and low-energy theorems (LETs) of the 1950's and 1960's [13,14, 15], which were based on current algebra and the PCAC hypothesis (partial conservation of the axial current). These relations become exact in the chiral limit of QCD, and thus all variables and observables have to be understood in the fictitious limit of vanishing (light) quark masses and hence soft pions with mass $M_{\pi} \rightarrow 0$. In this limit the threshold for pion photoproduction moves to the origin of the Mandelstam plane $(\nu=0, t=0)$. However, extensive investigations in ChPT [16,17, 18, 19 have shown that the finite pion mass leads to substantial corrections at physical threshold $\left(\nu=\nu_{\text {thr }} \approx 0.136 \mathrm{GeV}\right.$, $t=t_{\mathrm{thr}} \approx-0.016 \mathrm{GeV}^{2}$ ) and that even a previously derived LET for neutral pion photoproduction had to be corrected because of the non-analytic structure of the expansion coefficients in $M_{\pi}$. We have studied this aspect in the context of the sum rule of Fubini, Furlan, and Rossetti (FFR) [20, which relates the nucleon's anomalous magnetic moment $\kappa_{N}$ to a dispersion integral over the first pion photoproduction amplitude $A_{1}(\nu, t)$. Generalized to electroproduction, this integral takes the form

$$
\begin{aligned}
F_{2}^{N}\left(Q^{2}\right) & \tau_{3}+\Delta_{1}^{N}\left(\nu, t_{\mathrm{thr}}, Q^{2}\right)= \\
& \frac{4 M_{N}^{2}}{\pi e g_{\pi N}} \mathcal{P} \int_{\nu_{\mathrm{thr}}}^{\infty} \mathrm{d} \nu^{\prime} \frac{\nu^{\prime} \operatorname{Im} A_{1}^{\left(N, \pi^{0}\right)}\left(\nu^{\prime}, t_{\mathrm{thr}}, Q^{2}\right)}{\nu^{\prime 2}-\nu^{2}},
\end{aligned}
$$

where $F_{2}^{N}\left(Q^{2}\right)$ is the Pauli form factor normalized to $F_{2}^{N}(0)=\kappa_{N}$. Furthermore, $M_{N}$ is the mass of the nucleon, $g_{\pi N}$ the pion-nucleon coupling constant, and $e$ the elementary charge. Plotted as function of $\nu$ and at $Q^{2}=0$, the right-hand side (rhs) of Eq. (1) yields a pronounced Wigner cusp with a maximum of about 2.5 for the proton, about $50 \%$ higher than the anomalous magnetic moment $\kappa_{p}$. The origin of this cusp is the strong $\left(\pi^{+}, \pi^{0}\right)$ rescattering leading to a large imaginary 
B. Pasquini et al.: Invariant Amplitudes for Pion Electroproduction

part of the S-wave multipole, which opens like a square root and therefore yields a singularity of the integrand at the charged pion threshold. If however the integral is evaluated at $\nu \approx 0$, the loop effect at threshold is no longer enhanced, and the bulk contribution of the integral stems from the resonance region, in particular from the $N \rightarrow \Delta(1232)$ transition. Although a decrease of the integral for $\nu \rightarrow 0$ is therefore expected, we were surprised that near the origin of the Mandelstam plane the "FFR discrepancy" $\Delta_{1}^{N}(\nu, t, 0)$ is actually compatible with zero, in agreement with the FFR sum rule. However, this sum rule is derived for a world of massless pions, which would not only lower the threshold to zero but also change the anomalous magnetic moment and the absorptive spectrum.

In the present contribution we extend our work to the electroproduction of pions, which involves two additional longitudinal amplitudes and one additional variable, the virtuality $Q^{2}$ of the exchanged photon. Moreover, the threshold now depends on $Q^{2}$, i.e., $\nu_{\text {thr }}=\nu_{\text {thr }}\left(Q^{2}\right)$ and $t_{\mathrm{thr}}=t_{\mathrm{thr}}\left(Q^{2}\right)$. As in the real photon case, the threshold region opens a wide field of comparisons with recent experiments and predictions of baryon ChPT. For example, the loop corrections of ChPT have a very distinct $Q^{2}$ dependence [8], completely different from the form factors in the pole contributions, and some of the new experimental data still offer problems for the theoretical description. As a first step towards a new dispersive approach, we address two sum rules for virtual photons. The first one is given by Eq. (1) as function of $Q^{2}$. The second sum rule connects the axial $\left(G_{A}^{V}\right)$ and Dirac $\left(F_{1}^{V}\right)$ isovector form factors to the longitudinal amplitude $A_{6}$ with isospin (-). Its physics content is identical with the LET of Nambu et al. [21, which has been derived for the slope of the S-wave multipole. In the notation of Fubini et al. 20] this sum rule may be cast into the form

$$
\begin{aligned}
G_{A}^{V}\left(Q^{2}\right)- & F_{1}^{V}\left(Q^{2}\right)+\Delta_{6}^{(-)}\left(\nu, t_{\mathrm{thr}}, Q^{2}\right)= \\
& \frac{4 M_{N} Q^{2}}{\pi e g_{\pi N}} \mathcal{P} \int_{\nu_{\mathrm{thr}}}^{\infty} \mathrm{d} \nu^{\prime} \frac{\nu^{\prime} \operatorname{Im} A_{6}^{(-)}\left(\nu^{\prime}, t_{\mathrm{thr}}, Q^{2}\right)}{\nu^{\prime 2}-\nu^{2}}
\end{aligned}
$$

As in the former case, this sum rule is derived in the soft-pion limit, for which both the kinematic variables and the observables (form factors, multipoles) differ from the physical ones by terms $\mathcal{O}\left(M_{\pi}\right)$. In the limit $Q^{2} \rightarrow 0$, the lhs of Eq. (2) yields information on $\left\langle r^{2}\right\rangle_{1}^{V}-\left\langle r^{2}\right\rangle_{A}^{V}$, where $\left\langle r^{2}\right\rangle_{i}^{V}$ are the squares of the respective rootmean-square (r.m.s.) radii. The integral on the rhs now involves longitudinal multipoles also at $Q^{2}=0$, which requires an extrapolation from the measured values at finite $Q^{2}$. Moreover, the two radii are of similar size, and the first estimates 22 simply led to the result $G_{A}^{V}\left(Q^{2}\right)-F_{1}^{V}\left(Q^{2}\right)=0$. The first and to our knowledge only dispersive calculation of Eq. (2) was performed by Adler and Gilman already in 1966 [23. The result was $\left\langle r^{2}\right\rangle_{1}^{V}-\left\langle r^{2}\right\rangle_{A}^{V}=0.152 \mathrm{fm}^{2}$, in fantastic agreement with our present knowledge of this observable, $(0.14 \pm 0.03) \mathrm{fm}^{2}$ ! Unfortunately, it has to be realized that the multipoles used in performing the integral had large error bars. In particular, the longitudinal and transverse multipoles were assumed to be equal, which is only correct for the unphysical kinematics of the Siegert limit. As an example, the longitudinal and transverse S-wave multipoles take the same value in that limit, but already at $Q^{2}=0$ they are quite different, and in the resonance region even the relative sign between the longitudinal and the respective transverse multipoles may differ from the low-energy limit. However, the real merit of this early work is the observation that formidable cancelations occur (I) among contributions of positive sign in the region up to the $\Delta(1232)$ resonance and of negative sign in the second resonance region and (II) between the electric transverse and longitudinal contributions of the same multipolarity.

We proceed by summarizing the kinematics for pion electroproduction in Sec. 2, and in Sec. 3 we introduce the invariant and CGLN amplitudes. The status of the LETs and sum rules at finite $Q^{2}$ is discussed in Sec. 4. We present the predictions of dispersion theory in Sec. 5 and close by a short summary in Sec. 6 .

\section{Kinematics}

Let $p_{i}^{\mu}$ and $p_{f}^{\mu}$ be the four-momenta of the initial and final nucleons, and $k^{\mu}$ and $q^{\mu}$ the four-momenta of the photon and pion, respectively. In the c.m. system, we define

$$
\begin{aligned}
p_{i}^{\mu} & =\left(E_{i},-\mathbf{k}\right), \quad p_{f}^{\mu}=\left(E_{f},-\mathbf{q}\right), \\
k^{\mu} & =\left(k_{0}, \mathbf{k}\right), \quad q^{\mu}=\left(q_{0}, \mathbf{q}\right) .
\end{aligned}
$$

The familiar Mandelstam variables are

$$
s=\left(p_{i}+k\right)^{2}, \quad t=(q-k)^{2}, \quad u=\left(p_{i}-q\right)^{2},
$$

and $\nu=(s-u) /\left(4 M_{N}\right)$ is the crossing symmetric variable. This variable is related to the photon lab energy $E_{\gamma}^{\text {lab }}$ by $\nu=E_{\gamma}^{\mathrm{lab}}+\left(t-M_{\pi}^{2}+Q^{2}\right) /\left(4 M_{N}\right)$. The physical $s$-channel region is shown in Fig. 1 for $Q^{2}=0.1 \mathrm{GeV}^{2}$. Its upper and lower boundaries are given by the scattering angles $\theta=0$ and $\theta=180^{\circ}$, respectively. The nucleon and pion poles lie in the unphysical region and are indicated by the dotted lines at $\nu_{s}=\nu_{B}(\mathrm{~s}-$ channel $)$ and $\nu_{u}=-\nu_{B}(\mathrm{u}$-channel $)$, where

$$
\nu_{B}=\frac{t-M_{\pi}^{2}+Q^{2}}{\left(4 M_{N}\right)} .
$$

The threshold for pion electroproduction lies at

$$
\begin{aligned}
\nu_{\mathrm{thr}} & =\frac{M_{\pi}\left[\left(2 M_{N}+M_{\pi}\right)^{2}+Q^{2}\right]}{4 M_{N}\left(M_{N}+M_{\pi}\right)}, \\
t_{\mathrm{thr}} & =-\frac{M_{N}\left(M_{\pi}^{2}+Q^{2}\right)}{M_{N}+M_{\pi}},
\end{aligned}
$$

and the energies and momenta of the particles are given by

$$
k_{0}=\frac{s-Q^{2}-M_{N}^{2}}{2 \sqrt{s}}, \quad q_{0}=\frac{s+M_{\pi}^{2}-M_{N}^{2}}{2 \sqrt{s}}
$$




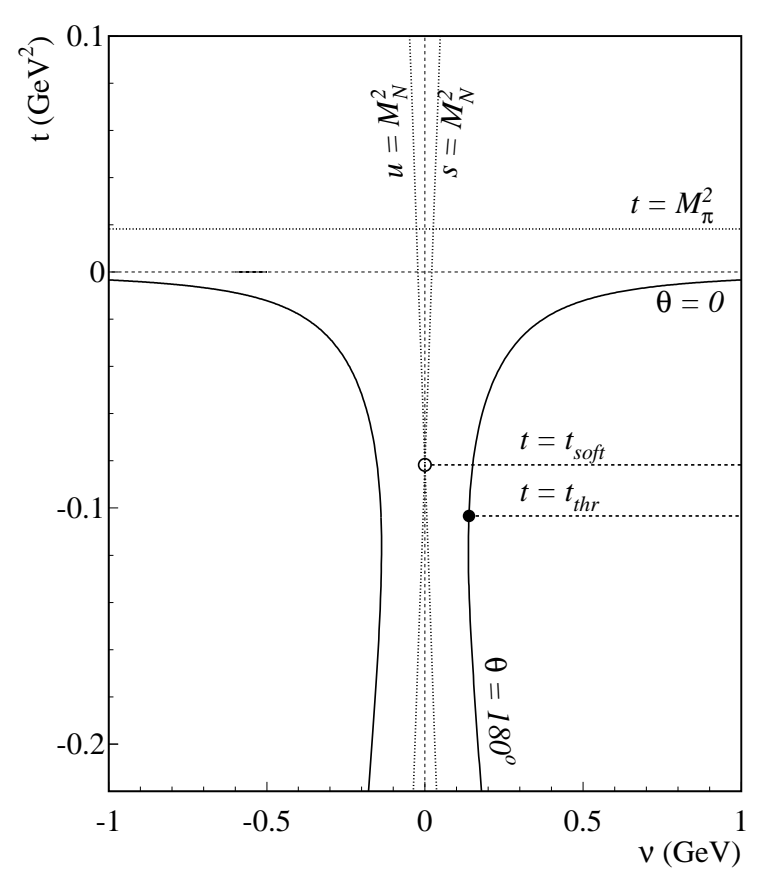

Figure 1. The Mandelstam plane for pion electroproduction on the nucleon. The solid line shows the boundary of the physical region for $Q^{2}=0.1 \mathrm{GeV}^{2}$. This boundary corresponds to forward production $\left(\theta=0^{\circ}\right)$ for $t \geq t_{\text {thr }}$ and to backward production $\left(\theta=180^{\circ}\right)$ for $t \leq t_{\mathrm{thr}}$. The nucleon and pion pole positions are indicated by the dotted lines $s=M_{N}^{2}, u=M_{N}^{2}$, and $t=M_{\pi}^{2}$. The threshold of pion electroproduction is represented by the solid circle, in the soft-pion limit the threshold moves to $\nu=\nu_{B}=0$ (open circle).

$$
\begin{aligned}
q & =|\mathbf{q}|=\left[\left(\frac{s+M_{\pi}^{2}-M_{N}^{2}}{2 \sqrt{s}}\right)^{2}-M_{\pi}^{2}\right]^{1 / 2}, \\
k & =|\mathbf{k}|=\left[\left(\frac{s-Q^{2}-M_{N}^{2}}{2 \sqrt{s}}\right)^{2}+Q^{2}\right]^{1 / 2}, \\
E_{i} & =W-k_{0}=\frac{s+M_{N}^{2}+Q^{2}}{2 \sqrt{s}}, \\
E_{f} & =W-q_{0}=\frac{s+M_{N}^{2}-M_{\pi}^{2}}{2 \sqrt{s}},
\end{aligned}
$$

with $W=\sqrt{s}$ the c.m. energy and $Q^{2}=-k^{\mu} k_{\mu}$. The pseudothreshold or Siegert limit for electroproduction lies at the unphysical point $\mathbf{k}=0$, which corresponds to $Q^{2}=$ $-\left(W-M_{N}\right)^{2}$.

\section{Invariant and CGLN Amplitudes}

The electromagnetic transition can be described by 6 invariant amplitudes $A_{i}$,

$$
\varepsilon_{\mu} J^{\mu}=\sum_{i=1}^{6} A_{i}\left(\nu, t, Q^{2}\right) \varepsilon_{\mu} M_{i}^{\mu},
$$

with $\varepsilon_{\mu}$ the polarization four-vector of the virtual photon and $J_{\mu}$ the transition current of the nucleon. In the notation of Dennery [3], the four-vectors $M_{i}^{\mu}$ take the following form :

$$
\begin{aligned}
M_{1}^{\mu} & =-\frac{1}{2} i \gamma_{5}\left(\gamma^{\mu} \not k-\not k \gamma^{\mu}\right), \\
M_{2}^{\mu} & =2 i \gamma_{5}\left(P^{\mu} k \cdot\left(q-\frac{1}{2} k\right)-\left(q-\frac{1}{2} k\right)^{\mu} k \cdot P\right), \\
M_{3}^{\mu} & =-i \gamma_{5}\left(\gamma^{\mu} k \cdot q-\not k q^{\mu}\right), \\
M_{4}^{\mu} & =-2 i \gamma_{5}\left(\gamma^{\mu} k \cdot P-\not k P^{\mu}\right)-2 M_{N} M_{1}^{\mu}, \\
M_{5}^{\mu} & =i \gamma_{5}\left(k^{\mu} k \cdot q+Q^{2} q^{\mu}\right), \\
M_{6}^{\mu} & =-i \gamma_{5}\left(\not k k^{\mu}+Q^{2} \gamma^{\mu}\right),
\end{aligned}
$$

with $P^{\mu}=\left(p_{i}+p_{f}\right)^{\mu} / 2, \phi=a_{\mu} \gamma^{\mu}$, and $\gamma$ matrices as defined in Ref. 24. In the case of real photons $\left(Q^{2}=0\right)$ and with the gauge condition $\varepsilon_{\mu} k^{\mu}=0$, the matrices $M_{5}^{\mu}$ and $M_{6}^{\mu}$ do not contribute to the interaction Lagrangian, and the remaining four matrices reduce to Eq. (10) of Ref. [1]. The invariant amplitudes $A_{i}$ can be further decomposed into three isospin channels $(a=1,2,3)$,

$$
A_{i}^{a}=A_{i}^{(-)} i \epsilon^{a 3 b} \tau^{b}+A_{i}^{(0)} \tau^{a}+A_{i}^{(+)} \delta_{a 3},
$$

where $\tau^{a}$ are the Pauli matrices in isospace, and the physical photoproduction amplitudes are given by

$$
\begin{aligned}
A_{i}\left(\gamma p \rightarrow n \pi^{+}\right) & =\sqrt{2}\left(A_{i}^{(-)}+A_{i}^{(0)}\right), \\
A_{i}\left(\gamma p \rightarrow p \pi^{0}\right) & =A_{i}^{(+)}+A_{i}^{(0)}, \\
A_{i}\left(\gamma n \rightarrow p \pi^{-}\right) & =-\sqrt{2}\left(A_{i}^{(-)}-A_{i}^{(0)}\right), \\
A_{i}\left(\gamma n \rightarrow n \pi^{0}\right) & =A_{i}^{(+)}-A_{i}^{(0)} .
\end{aligned}
$$

Under crossing, the amplitudes $A_{1,2,4}^{(+, 0)}$ and $A_{3,5,6}^{(-)}$are even functions of $\nu$ and satisfy a DR of the type

$$
\begin{gathered}
A_{i, \text { disp }}^{(I)}\left(\nu, t, Q^{2}\right) \equiv \operatorname{Re} A_{i}^{(I)}\left(\nu, t, Q^{2}\right)-A_{i, \text { pole }}^{(I)}\left(\nu, t, Q^{2}\right) \\
\quad=\frac{2}{\pi} \mathcal{P} \int_{\nu_{t h r}}^{\infty} \mathrm{d} \nu^{\prime} \frac{\nu^{\prime} \operatorname{Im} A_{i}^{(I)}\left(\nu^{\prime}, t, Q^{2}\right)}{\nu^{\prime 2}-\nu^{2}}
\end{gathered}
$$

whereas the amplitudes $A_{3,5,6}^{(+, 0)}$ and $A_{1,2,4}^{(-)}$are odd and therefore fulfil the relation

$$
A_{i, \text { disp }}^{(I)}\left(\nu, t, Q^{2}\right)=\frac{2 \nu}{\pi} \mathcal{P} \int_{\nu_{\text {thr }}}^{\infty} \mathrm{d} \nu^{\prime} \frac{\operatorname{Im} A_{i}^{(I)}\left(\nu^{\prime}, t, Q^{2}\right)}{\nu^{\prime 2}-\nu^{2}} .
$$

The nucleon pole contributions $A_{i, \text { pole }}^{(I)}$ can be written as functions of the Mandelstam variables and $Q^{2}$ as follows:

$$
\begin{aligned}
& A_{1, \text { pole }}^{(I)}=\frac{e g_{\pi N}}{2}\left(\frac{1}{s-M_{N}^{2}}+\frac{\epsilon^{I}}{u-M_{N}^{2}}\right) F_{1}^{(I)}\left(Q^{2}\right) \\
& A_{2, \text { pole }}^{(I)}=-\frac{e g_{\pi N}}{t-M_{\pi}^{2}}\left(\frac{1}{s-M_{N}^{2}}+\frac{\epsilon^{I}}{u-M_{N}^{2}}\right) F_{1}^{(I)}\left(Q^{2}\right) \\
& A_{3, \text { pole }}^{(I)}=-\frac{e g_{\pi N}}{4 M_{N}}\left(\frac{1}{s-M_{N}^{2}}-\frac{\epsilon^{I}}{u-M_{N}^{2}}\right) F_{2}^{(I)}\left(Q^{2}\right),
\end{aligned}
$$




$$
\begin{aligned}
A_{4, \text { pole }}^{(I)}= & -\frac{e g_{\pi N}}{4 M_{N}}\left(\frac{1}{s-M_{N}^{2}}+\frac{\epsilon^{I}}{u-M_{N}^{2}}\right) F_{2}^{(I)}\left(Q^{2}\right), \\
A_{5, \text { pole }}^{(I)}= & -\frac{e g_{\pi N}}{2\left(t-M_{\pi}^{2}\right)}\left(\frac{1}{s-M_{N}^{2}}-\frac{\epsilon^{I}}{u-M_{N}^{2}}\right) F_{1}^{(I)}\left(Q^{2}\right) \\
& +\frac{2 e g_{\pi N}\left[F_{\pi}^{V}\left(Q^{2}\right)-F_{1}^{(-)}\left(Q^{2}\right)\right]}{Q^{2}\left(t-M_{\pi}^{2}\right)} \delta_{I,-}, \\
A_{6, \text { pole }}^{(I)}= & 0,
\end{aligned}
$$

with $\epsilon^{+}=\epsilon^{0}=-\epsilon^{-}=1, F_{i}^{(0)}=F_{i}^{p}+F_{i}^{n}=F_{i}^{S}$ the isoscalar and $F_{i}^{(+,-)}=F_{i}^{p}-F_{i}^{n}=F_{i}^{V}$ the isovector form factors, normalized to $F_{1}^{I}(0)=1, F_{2}^{(0)}=\kappa_{p}+\kappa_{n}$, and $F_{2}^{(+,-)}=\kappa_{p}-\kappa_{n}$, where $\kappa_{p}$ and $\kappa_{n}$ are the anomalous magnetic moments of proton and neutron, respectively.

For further use we also list the contributions of $t$ channel vector-meson exchange to the isospin $(+)$ and $(0)$ amplitudes :

$$
\begin{aligned}
& A_{1}^{(+, 0)}\left(t, Q^{2}\right)=\frac{e \lambda_{V} g_{V}^{(T)}}{2 M_{N} M_{\pi}} \frac{t}{t-m_{V}^{2}} F_{\gamma \pi V}\left(Q^{2}\right), \\
& A_{2}^{(+, 0)}\left(t, Q^{2}\right)=-\frac{e \lambda_{V} g_{V}^{(T)}}{2 M_{N} M_{\pi}} \frac{t-M_{\pi}^{2}-Q^{2}}{\left(t-m_{V}^{2}\right)\left(t-M_{\pi}^{2}\right)} F_{\gamma \pi V}\left(Q^{2}\right), \\
& A_{3}^{(+, 0)}\left(t, Q^{2}\right)=0, \\
& A_{4}^{(+, 0)}\left(t, Q^{2}\right)=-\frac{e \lambda_{V} g_{V}^{(V)}}{M_{\pi}} \frac{1}{t-m_{V}^{2}} F_{\gamma \pi V}\left(Q^{2}\right), \\
& A_{5}^{(+, 0)}\left(t, Q^{2}\right)=\frac{e \lambda_{V} g_{V}^{(T)}}{M_{\pi}} \frac{\nu}{\left(t-m_{V}^{2}\right)\left(t-M_{\pi}^{2}\right)} F_{\gamma \pi V}\left(Q^{2}\right), \\
& A_{6}^{(+, 0)}\left(t, Q^{2}\right)=0,
\end{aligned}
$$

where $\lambda_{V}$ denotes the coupling of the vector meson $(V=\omega, \rho)$ to the $\gamma \pi$ system, $g_{V}^{(V, T)}$ its vector or tensor coupling to the nucleon, and $F_{\gamma \pi V}\left(Q^{2}\right)$ is a transition form factor. For further details see Ref. [2].

The matrix element of the electromagnetic current, Eq. (8), takes the form

$$
\bar{u}\left(p_{f}\right) \sum_{i=1}^{6} A_{i} \varepsilon_{\mu} M_{i}^{\mu} u\left(p_{i}\right)=\frac{4 \pi W}{M_{N}} \chi_{f}^{\dagger} \mathcal{F} \chi_{i}
$$

with $u(p)$ the Dirac spinor of the nucleon with $\bar{u}(p) u(p)=$ $2 M_{N}$, and $\chi$ the Pauli spinor of the nucleon. The operator $\mathcal{F}$ in Eq. (16) can be decomposed into the CGLN amplitudes $\mathcal{F}_{i}$ [25,

$$
\begin{array}{cc}
\mathcal{F}= & -i(\boldsymbol{\sigma} \cdot \mathbf{b}) \mathcal{F}_{1}-(\boldsymbol{\sigma} \cdot \hat{\mathbf{q}}) \mathbf{b} \cdot(\boldsymbol{\sigma} \times \hat{\mathbf{k}}) \mathcal{F}_{2}- \\
& i \quad(\mathbf{b} \cdot \hat{\mathbf{q}})(\boldsymbol{\sigma} \cdot \hat{\mathbf{k}}) \mathcal{F}_{3}-i(\mathbf{b} \cdot \hat{\mathbf{q}})(\boldsymbol{\sigma} \cdot \hat{\mathbf{q}}) \mathcal{F}_{4}+ \\
& i \quad(\boldsymbol{\sigma} \cdot \hat{\mathbf{k}}) b_{0} \frac{k}{k_{0}} \mathcal{F}_{5}+i(\boldsymbol{\sigma} \cdot \hat{\mathbf{q}}) b_{0} \frac{k}{k_{0}} \mathcal{F}_{6},
\end{array}
$$

where $b^{\mu}=\varepsilon^{\mu}-(\boldsymbol{\epsilon} \cdot \hat{\mathbf{k}}) k^{\mu} / k$. The relations between the invariant amplitudes $A_{i}$ and the CGLN amplitudes $\mathcal{F}_{i}$ are obtained by combining Eqs. (16) and (17). The general result is given in Appendix $\mathrm{A}$ and the multipole series for the CGLN amplitudes is shown in Appendix B. Specifically, the invariant amplitude $A_{1}$ at threshold has the following multipole decomposition:

$$
\begin{aligned}
A_{1}^{\mathrm{thr}} & =\frac{4 \pi}{M_{N}\left(\mu^{2}+\rho\right) \sqrt{(1+\mu)\left[(2+\mu)^{2}+\rho\right]}} \\
& \times\left\{(1+\mu)[\mu(2+\mu)+\rho] E_{0+}+\frac{4(1+\mu)^{2} \rho}{\mu(2+\mu)-\rho} L_{0+}\right. \\
& -M_{N} \mu \sqrt{\left(\mu^{2}+\rho\right)\left[(2+\mu)^{2}+\rho\right]} \bar{P}_{2} \\
& -\frac{M_{N}(2+\mu)\left(\mu^{2}+\rho\right)^{3 / 2}}{\sqrt{(2+\mu)^{2}+\rho}} \bar{P}_{3}-2 M_{N}^{2} \mu\left(\mu^{2}+\rho\right) \bar{D} \\
& \left.+\frac{8 M_{N}(1+\mu)^{2} \rho \sqrt{\mu^{2}+\rho}}{[\mu(2+\mu)-\rho] \sqrt{(2+\mu)^{2}+\rho}} \bar{P}_{5}\right\},
\end{aligned}
$$

where we have introduced the ratios $\mu=M_{\pi} / M_{N}$ and $\rho=Q^{2} / M_{N}^{2}$, and the following combinations of the Pand D-wave multipoles:

$$
\begin{aligned}
\bar{P}_{2} & =\left(3 E_{1+}-M_{1+}+M_{1-}\right) / q, \\
\bar{P}_{3} & =\left(2 M_{1+}+M_{1-}\right) / q, \\
\bar{P}_{5} & =\left(L_{1-}-2 L_{1+}\right) / q, \\
\bar{D} & =3\left(M_{2+}-E_{2+}-M_{2-}-E_{2-}\right) / q^{2} .
\end{aligned}
$$

We note that all the multipoles in Eqs. (18) to (20) should be evaluated at $W_{\mathrm{thr}}=M_{N}(1+\mu)$. The factor $\left(\mu^{2}+\rho\right)^{-1}$ in Eq. (18) is worrying because of the singularity in the Siegert limit $k \rightarrow 0$, which corresponds to $Q^{2} \rightarrow-M_{\pi}^{2}$ or $\rho \rightarrow-\mu^{2}$ in our notation. We may eliminate this critical factor by use of the Siegert limit for the multipoles (see Appendix (B). In particular, the S-wave multipoles are even functions of $k$, and since both $L_{0+}$ and $E_{0+}$ approach the same constant in that limit, the difference of the two multipoles is proportional to $k^{2}$. Furthermore, all the $\mathrm{P}$-waves contain a factor $k$. With the definitions $\Delta_{0+}=\left(L_{0+}-E_{0+}\right) / k^{2}$ and $\mathcal{P}_{i}=P_{i} /(q k)=\bar{P}_{i} / k$ we can rewrite Eq. (18) as follows:

$$
\begin{aligned}
A_{1}^{\text {thr }} & =\frac{4 \pi M_{N}}{\sqrt{(1+\mu)\left[(2+\mu)^{2}+\rho\right]}} \\
& \times\left\{\frac{(1+\mu)\left[(2+\mu)^{2}-\rho\right]}{M_{N}^{2}(\mu(2+\mu)-\rho)} E_{0+}+\frac{\left[(2+\mu)^{2}+\rho\right] \rho}{\mu(2+\mu)-\rho} \Delta_{0+}\right. \\
& -\frac{\mu\left[(2+\mu)^{2}+\rho\right]}{2(1+\mu)} \mathcal{P}_{2}-\frac{(2+\mu)\left(\mu^{2}+\rho\right)}{2(1+\mu)} \mathcal{P}_{3} \\
& \left.-2 \mu \bar{D}+\frac{4(1+\mu) \rho}{\mu(2+\mu)-\rho} \mathcal{P}_{5}\right\} .
\end{aligned}
$$

Note that we have left the term $\bar{D}$ unchanged, because only the first three D waves in Eq. (19) are proportional to $k^{2}$ in the Siegert limit, whereas $E_{2-}$ is an electric dipole transition and therefore approaches a constant in that limit. Comparing now Eq. (18) with Eq. (20), we find that the pole at $\left(\mu^{2}+\rho\right)$ has disappeared in the latter equation. The remaining kinematical pole at $\mu(2+\mu)-\rho$ 
is compensated by a zero in the longitudinal multipoles. The corresponding equations for the longitudinal amplitudes $A_{5}$ and $A_{6}$ are given in Appendix C.

It is evident from Eq. (9) that the 6 "Dennery amplitudes" $A_{i}$ fulfill gauge invariance. However, Eqs. (50) and (53) of Appendix $\mathrm{A}$ show that the amplitudes $A_{2}$ and $A_{5}$ have kinematical singularities at $t=M_{\pi}^{2}$. These singularities can be avoided by introducing a set of 8 four-vectors $N_{i}^{\mu}$ 4, which are free of kinematical singularities and therefore should obey a Mandelstam representation. However, these amplitudes are not separately gauge invariant. In order to implement gauge invariance, the associated "Ball amplitudes" $B_{i}$ have to fulfill two additional constraints 4. As discussed in more detail by v. Gehlen 6, these constraints lead to an additional kinematical singularity at the pion pole $\left(t=M_{\pi}^{2}\right)$, which should not contribute to the residue of (I) $A_{2}$ for all values of $\nu$ and real photons $\left(Q^{2}=0\right)$, and (II) $A_{5}$ for $\nu=0$ and all values of $Q^{2}$. This has no further consequences for the function $A_{2}$. However, the insertion of the second condition in Eq. (12) requires that

$$
\operatorname{Re} A_{5, \operatorname{disp}}^{(-)}\left(0, t, Q^{2}\right)=\frac{2}{\pi} \int_{\nu_{t h r}}^{\infty} \frac{\mathrm{d} \nu^{\prime}}{\nu^{\prime}} \operatorname{Im} A_{5}^{(-)}\left(\nu^{\prime}, t, Q^{2}\right) .
$$

Since the invariant amplitudes should only have a dynamical singularity in $t$, namely the pion pole term, any contribution of the dispersive integral with the behavior of the pion pole term has to be subtracted, i.e., Eq. (12) has to be corrected by

$$
\begin{aligned}
& \operatorname{Re} A_{5}^{(-)}\left(\nu, t, Q^{2}\right) \rightarrow \operatorname{Re} A_{5}^{(-)}\left(\nu, t, Q^{2}\right)- \\
& \frac{2}{\pi} \frac{1}{t-M_{\pi}^{2}} \int_{\nu_{\mathrm{thr}}}^{\infty} \frac{\mathrm{d} \nu^{\prime}}{\nu^{\prime}} \lim _{t^{\prime} \rightarrow M_{\pi}^{2}}\left\{\left(t^{\prime}-M_{\pi}^{2}\right) \operatorname{Im} A_{5}^{(-)}\left(\nu^{\prime}, t^{\prime}, Q^{2}\right)\right\}
\end{aligned}
$$

As a result the corrected dispersion integral does no longer contribute to the pion-pole residue. In particular at $\nu=0$, this residue is given by the pole contribution of Eq. (14) as follows:

$$
A_{5, \text { pole }}^{(-)}\left(0, t, Q^{2}\right)=\frac{2 e g_{\pi N}}{Q^{2}}\left\{\frac{F_{\pi}^{V}\left(Q^{2}\right)}{t-M_{\pi}^{2}}-\frac{F_{1}^{V}\left(Q^{2}\right)}{t-M_{\pi}^{2}+Q^{2}}\right\} .
$$

\section{Low Energy Theorems and Sum Rules}

Several LETs for pion photo- and electroproduction were derived in the 1950's and 1960's from PCAC and current algebra which preceded QCD. A modern framework to derive these sum rules is provided by ChPT as an effective realization of QCD in terms of its low-energy degrees of freedom. The first of these theorems is due to Kroll and Ruderman [13] who found that the threshold photoproduction of charged pions is described by minimal coupling of the photon to the pseudovector pion-nucleon interaction. Nambu and collaborators [21] extended these considerations to virtual photons and obtained a relation between the S-wave multipole for pion electroproduction with isospin $(-)$ and the difference of the isovector Dirac and axial form factors. Finally Fubini, Furlan, and Rossetti [20] derived a sum rule for the Pauli form factors in terms of the amplitudes $A_{1}^{(+, 0)}$. The origin of these sum rules is summarized as follows:

(I) The electromagnetic transition current $J_{\mu}$ can be expanded in the set of the 6 covariants $M_{i}^{\mu}$, see Eq. (9). In the limit of vanishing pion four-momentum only the covariants $M_{1}^{\mu}$ and $M_{6}^{\mu}$ survive, and therefore the softpion transition current is completely determined by the associated invariant amplitudes $A_{1}$ and $A_{6}$.

(II) The soft-pion limit $q_{\mu} \rightarrow 0$ is obtained by first going to threshold ( $\mathbf{q}=0, q_{0}=M_{\pi}$ in the hadronic c.m. system) and then removing the mass of the pion $\left(q_{0}=\right.$ $\left.M_{\pi} \rightarrow 0\right)$. The second step requires an extrapolation into unphysical territory, which can only be performed within a theoretical framework.

In the following we consider the dispersive contribution to threshold amplitudes, $A_{i \text {, disp }}^{(I) \mathrm{thr}} \equiv A_{i, \mathrm{disp}}^{(I)}\left(\nu_{\mathrm{thr}}, t_{\mathrm{thr}}, Q^{2}\right)$, where $\nu_{\mathrm{thr}}=\nu_{\mathrm{thr}}\left(Q^{2}\right)$ and $t_{\mathrm{thr}}=t_{\mathrm{thr}}\left(Q^{2}\right)$ are given by Eq. (6). According to Ref. 20, the crossing-even amplitudes $A_{1, \text { disp }}^{(+, 0) \text { thr }}$ and $A_{6 \text {, disp }}^{(-) \text {, thr }}$ take the following form in the soft-pion limit:

$$
\begin{aligned}
A_{1, \mathrm{disp}}^{(+, 0) \mathrm{thr}} & \longrightarrow \frac{e g_{\pi N}}{4 M_{N}^{2}} F_{2}^{V, S}\left(Q^{2}\right) \equiv A_{1, \mathrm{FFR}}^{(+, 0)}\left(Q^{2}\right) \\
A_{6, \mathrm{disp}}^{(-) \mathrm{thr}} & \longrightarrow \frac{e g_{\pi N}}{2 M_{N} Q^{2}}\left[G_{A}^{V}\left(Q^{2}\right)-F_{1}^{V}\left(Q^{2}\right)\right] \equiv A_{6, \mathrm{FFR}}^{(-)}\left(Q^{2}\right) \\
& =\frac{e g_{\pi N}}{12 M_{N}}\left(\left\langle r^{2}\right\rangle_{1}^{V}-\left\langle r^{2}\right\rangle_{A}^{V}\right)+\ldots
\end{aligned}
$$

whereas the crossing-odd amplitudes $A_{1}^{(-)}$and $A_{6}^{(+, 0)}$ vanish in that limit. We repeat that these results are only valid in the world of massless pions.

The corrections due to the finite mass of the pion have been calculated in ChPT. For further use we list the Swave multipoles at threshold as obtained to $\mathcal{O}\left(q^{2}\right)$ by manifestly Lorentz invariant baryon ChPT [8],

$$
\begin{aligned}
E_{0+}^{(+) \operatorname{thr}} & =x_{\pi N}\left\{-2 \mu+\left(3+\kappa_{V}\right) \mu^{2}+\left(1+\kappa_{V}\right) \rho\right. \\
& \left.+2 \mu^{2} y_{\pi N} \Xi_{1}\right\}, \\
L_{0+}^{(+) \mathrm{thr}} & =E_{0+}^{(+) \operatorname{thr}}+x_{\pi N}\left(\mu^{2}+\rho\right)\left\{-\kappa_{V}+2 y_{\pi N} \Xi_{2}\right\}, \\
E_{0+}^{(0) \mathrm{thr}} & =x_{\pi N}\left\{-2 \mu+\left(3+\kappa_{S}\right) \mu^{2}+\left(1+\kappa_{S}\right) \rho\right\}, \\
L_{0+}^{(0) \mathrm{thr}} & =E_{0+}^{(0) \mathrm{thr}}-x_{\pi N}\left(\mu^{2}+\rho\right) \kappa_{S}, \\
E_{0+}^{(-) \operatorname{thr}} & =4 x_{\pi N}\left\{1-\mu+\left(\frac{9}{8}+C_{0}\right) \mu^{2}\right. \\
& \left.-\frac{\rho}{4}\left[\kappa_{V}+\frac{1}{2}+\frac{2}{3} M_{N}^{2}\left\langle r^{2}\right\rangle_{A}^{V}\right]+\frac{\mu^{2} y_{\pi N}}{\pi} \Xi_{3}\right\},
\end{aligned}
$$




$$
\begin{aligned}
L_{0+}^{(-) \mathrm{thr}} & =E_{0+}^{(-) \mathrm{thr}}+x_{\pi N}\left(\mu^{2}+\rho\right)\left\{\kappa_{V}\right. \\
& -\frac{2 \sqrt{(2+\mu)^{2}+\rho}}{(1+\mu)^{3 / 2}\left[\mu^{2}(2+\mu)+\rho\right]}+\frac{2}{3} M_{N}^{2}\left\langle r^{2}\right\rangle_{A}^{V} \\
& \left.-\frac{2}{3} M_{N}^{2}\left[1-\frac{\rho}{2 \mu^{2}+\rho}\right]\left\langle r^{2}\right\rangle_{\pi}^{V}+\frac{4 y_{\pi N}}{\pi} \Xi_{4}\right\},
\end{aligned}
$$

with $C_{0} \approx-0.725$ as estimated by resonance saturation, $x_{\pi N}=e g_{\pi N} /\left(32 \pi M_{N}\right)$, and $y_{\pi N}=M_{N}^{2} /\left(8 \pi F_{\pi}^{2}\right)$. Furthermore, the loop functions $\Xi_{i}=\Xi_{i}\left(Q^{2} / M_{\pi}^{2}\right)$ can be expanded in a power series for $Q^{2} \ll M_{\pi}^{2}$,

$$
\begin{aligned}
& \Xi_{1}\left(Q^{2} / M_{\pi}^{2}\right)=\pi+\frac{Q^{2}}{2 M_{\pi}^{2}}(4-\pi)+\ldots, \\
& \Xi_{2}\left(Q^{2} / M_{\pi}^{2}\right)=(2-\pi)+\frac{2 Q^{2}}{M_{\pi}^{2}}(\pi-3)+\ldots, \\
& \Xi_{3}\left(Q^{2} / M_{\pi}^{2}\right)=\frac{\pi^{2}}{8}+\frac{1}{2}+\frac{Q^{2}}{16 M_{\pi}^{2}}\left(12-\pi^{2}\right)+\ldots, \\
& \Xi_{4}\left(Q^{2} / M_{\pi}^{2}\right)=\frac{1}{8}\left(4-\pi^{2}\right)+\frac{Q^{2}}{4 M_{\pi}^{2}}\left(\pi^{2}-9\right)+\ldots
\end{aligned}
$$

This expansion shows that the loop contributions increase with $Q^{2}$, whereas the form factors in the pole and FFR terms generally decrease with the virtuality of the photon. Although the loop effects of Eq. (26) are formally of $\mathcal{O}\left(q^{2}\right)$, they yield large corrections to the slopes in $Q^{2}$. In the case of neutral pion photoproduction, with isospin $(+)$ and $(0)$, these effects are even more important such that the LET of the 1960's had to be revised [16,7]. In fact, the lowest order loop corrections for neutral pion photoproduction have a larger absolute value than the leading term.

Equation (26) contains the pole terms (Appendix D), the FFR contributions (Appendix E), and in addition the loop and counter terms. In order to compare with the dispersion integrals, we have to subtract the pole terms. The results for the dispersive amplitudes take the following form at lowest order:

$$
\begin{aligned}
E_{0+, \mathrm{disp}}^{\left(p \pi^{0}\right) \mathrm{thr}} & =\frac{e g_{\pi N} M_{\pi}}{8 \pi M_{N}^{2}}\left\{\kappa_{p}\left(1-\frac{M_{\pi}}{M_{N}}\right)+\frac{M_{N} M_{\pi}}{16 \pi F_{\pi}^{2}} \Xi_{1}\right\} \\
& \approx\left(8.5+24.1 \frac{Q^{2}}{\mathrm{GeV}^{2}}\right) \frac{10^{-3}}{M_{\pi^{+}}} \\
L_{0+, \mathrm{disp}}^{\left(p \pi^{0}\right) \mathrm{thr}} & =E_{0+, \mathrm{disp}}^{\left(p \pi^{0}\right) \operatorname{thr}}+\frac{e g_{\pi N}}{16 \pi M_{N}^{3}}\left(M_{\pi}^{2}+Q^{2}\right) \\
& \times\left\{-\kappa_{p}+\frac{M_{N}^{2}}{8 \pi F_{\pi}^{2}} \Xi_{2}\right\} \\
& \approx\left(6.9-48.7 \frac{Q^{2}}{\mathrm{GeV}^{2}}\right), \frac{10^{-3}}{M_{\pi^{+}}} \\
E_{0+, \mathrm{disp}}^{(-) \mathrm{thr}} & =\frac{e g_{\pi N}}{8 \pi M_{N}^{3}}\left\{C_{0} M_{\pi}^{2}+\frac{M_{N}^{2} M_{\pi}^{2}}{8 \pi^{2} F_{\pi}^{2}} \Xi_{3}\right. \\
& \left.+\frac{M_{N}^{2} Q^{2}}{6}\left(\left\langle r^{2}\right\rangle_{1}^{V}-\left\langle r^{2}\right\rangle_{A}^{V}\right)\right\} \\
& \approx\left(0.8+19.4 \frac{Q^{2}}{\mathrm{GeV}^{2}}\right) \frac{10^{-3}}{M_{\pi+}}
\end{aligned}
$$

$$
\begin{aligned}
L_{0+, \mathrm{disp}}^{(-) \mathrm{thr}} & =E_{0+, \mathrm{disp}}^{(-) \operatorname{thr}}+\frac{e g_{\pi N}}{48 \pi M_{N}}\left(M_{\pi}^{2}+Q^{2}\right) \\
& \times\left\{\left\langle r^{2}\right\rangle_{A}^{V}-\left\langle r^{2}\right\rangle_{1}^{V}+\frac{3}{4 \pi^{2} F_{\pi}^{2}} \Xi_{4}\right\} \\
& \approx\left(0.03-13.7 \frac{Q^{2}}{\mathrm{GeV}^{2}}\right) \frac{10^{-3}}{M_{\pi^{+}}} .
\end{aligned}
$$

The numerical values in the above equations are obtained by a strict expansion to $\mathcal{O}\left(q^{2}\right)$ with the radii and other constants as given in Sec. 5. In order to judge the physical relevance of these leading order dispersive effects, they have to be compared to the corresponding pole contributions given by Appendix D.

$$
\begin{aligned}
E_{0+, \text { pole }}^{\left(p \pi^{0}\right) \mathrm{thr}} & =\left(-7.9+56.8 \frac{Q^{2}}{\mathrm{GeV}^{2}}\right) \frac{10^{-3}}{M_{\pi^{+}}}, \\
L_{0+, \text { pole }}^{\left(p \pi^{0}\right) \mathrm{thr}} & =\left(-7.9+55.7 \frac{Q^{2}}{\mathrm{GeV}^{2}}\right) \frac{10^{-3}}{M_{\pi^{+}}}, \\
E_{0+, \text { pole }}^{(-) \mathrm{thr}} & =\left(21.0-80.4 \frac{Q^{2}}{\mathrm{GeV}^{2}}\right) \frac{10^{-3}}{M_{\pi^{+}}}, \\
L_{0+, \text { pole }}^{(-) \mathrm{thr}} & =\left(11.9-300.0 \frac{Q^{2}}{\mathrm{GeV}^{2}}\right) \frac{10^{-3}}{M_{\pi^{+}}} .
\end{aligned}
$$

Let us first have a look at neutral pion electroproduction as described by Eqs. (28, 29) and (32, 33). It is seen that the dispersive and pole contributions cancel to about one order of magnitude at $Q^{2}=0$, which leads to very small physical photoproduction amplitudes at threshold. Also the slopes in $Q^{2}$ take large absolute values for both contributions. However, they add for the electric amplitude, whereas there is again a large cancelation for the longitudinal S-wave amplitude. Therefore, $L_{0+}^{\left(p \pi^{0}\right) \text { thr }}$ is significantly suppressed relative to the transverse S-wave multipole. Because the pole amplitude is well defined, we may conclude that the physically interesting dispersive amplitude can be well determined for both multipoles, although the respective cross sections are much smaller than expected from the size of the pole terms. The situation is quite different for the isospin (-) multipoles to be measured by charged pion production. Equations (30, 31) show that the dispersive effects are small compared to the pole contributions, Eqs. (34, 35). In particular in the real photon limit, the dispersive contributions are at most a few per cent of the respective pole contributions. The same is true for the slope of the longitudinal $\mathrm{S}$ wave, which has an extremely large pole contribution. In conclusion, the corresponding cross sections are large but the physically interesting dispersive contributions are hidden under the large "background" of the pole terms. The only exception is the slope of $E_{0+\text {,disp }}^{(-) \text {thr }}$ containing information on $\left\langle r^{2}\right\rangle_{1}^{V}-\left\langle r^{2}\right\rangle_{A}^{V}$, which takes the value $0.141 \mathrm{fm}^{2}$ with the radii given in Sect. 5 . However, as has been pointed out by Bernard et al. [18, the loop function $\Xi_{3}$ yields a sizeable loop correction of $0.046 \mathrm{fm}^{2}$. It is therefore mandatory to include the loop effects in the data analysis for pion electroproduction. One should also keep in mind that the experiment is dominated by the pole term of Eq. (34), that is, the dispersive contribution yields only about $10 \%$ of the total threshold 
multipole $E_{0+}^{(-)}$at $Q^{2}=0.05 \mathrm{GeV}^{2}$. In the real photon limit, the information on the radii is contained in the longitudinal S-wave multipole, $L_{0+\text {,pole }}^{(-) \text {thr }}$ The numerical value of the dispersive contribution, Eq. (31), is however dwarfed by the pole term, Eq. (35). We conclude that the longitudinal S-wave multipole has no practical relevance for studies of the axial radius, because the radius-dependent term and the loop corrections have different signs resulting in a very small net effect, the remaining small value is likely to change by higher-order loop effects, and the pole contribution is larger than the predicted dispersive effect by about two orders of magnitude. After this review of the key phenomena as predicted by ChPT, we present our dispersive results in the following section.

\section{Results and Discussion}

The numerical calculations in this section are performed with the following values: $g_{\pi N}=13.4, e=\sqrt{4 \pi / 137}, M_{N}=$ $0.938 \mathrm{GeV}, M_{\pi^{+}}=0.140 \mathrm{GeV}, M_{\pi^{0}}=0.135 \mathrm{GeV}, F_{\pi}=$ $0.0924 \mathrm{GeV}, \kappa_{p}=1.793$, and $\kappa_{n}=-1.913$.

\subsection{Dispersive contributions to the invariant amplitudes}

The dispersive contributions to the invariant amplitudes are shown as function of the crossing-symmetric variable $\nu$ in Fig. 2 for the $\mathrm{p} \pi^{0}$ and isospin $(-)$ channels, respectively. Even though all calculations are isospin symmetric, we define the threshold as $W=M_{p}+M_{\pi^{0}}$ for the isospin $(+, 0)$ channels and $W=M_{n}+M_{\pi^{+}}$for the $(-)$ channel. The Wigner cusp at the onset of charged pion production is clearly seen for the amplitudes $A_{1}$ and $A_{6}$, which receive large contributions from the multipoles $E_{0+}$ and $L_{0+}$. Comparing the results for real photons (solid lines, $Q^{2}=0$ ) and virtual photons (dashed lines, $Q^{2}=0.1 \mathrm{GeV}^{2}$ ), we find strong differences for some of the amplitudes over this moderate range of the virtuality. As has been shown in our previous work [1,2, the pole contributions due to the $t$-channel exchange of vector mesons must be included explicitly (see the dotted lines) in order to agree with the data for neutral pion production. For charged pion production, it is not possible to construct the dispersive amplitudes directly from the threshold data in a reliable way, because the pole contributions dominate this region. The contributions to the dispersion integral from the S, P, and higher multipoles are shown in Fig. 3 . It is seen that the S-wave contribution is strong for the real part of the threshold amplitudes $A_{1}$ and $A_{6}$, whereas all the other amplitudes are dominated by the $\mathrm{P}$ waves in the imaginary part of the amplitudes. The following Fig. 4 displays the dispersive contributions to the invariant amplitudes as function of the virtuality $Q^{2}$. In most cases the amplitudes change rather dramatically over the range $0 \leq Q^{2} \leq 0.1 \mathrm{GeV}^{2}$.

\subsection{The FFR sum rule for the invariant amplitude $A_{1}$}

For small values of the variables, the dispersive part of the invariant amplitudes can be expanded in a Taylor series. As an example, we cast Eq. (1) into the form

$$
A_{1, \mathrm{disp}}^{\left(p \pi^{0}\right)}\left(\nu, \nu_{B}, Q^{2}\right)=\frac{e g_{\pi N}}{2 M_{N}^{2}}\left(F_{2}^{p}\left(Q^{2}\right)+\Delta\left(\nu, \nu_{B}, Q^{2}\right)\right)
$$

with $\Delta \equiv \Delta_{1}^{\left(p \pi^{0}\right)}$ the dimensionless "FFR discrepancy". This function is real in a triangle defined by the straight lines $s=\left(M_{N}+M_{\pi}\right)^{2}, u=\left(M_{N}+M_{\pi}\right)^{2}$, and $t=4 M_{\pi}^{2}$, which define the onset of particle production in the $s, u$, and $t$ channels, respectively. In this region of the Mandelstam plane, the crossing-even function $\Delta_{1}^{\left(p \pi^{0}\right)}$ has the expansion

$$
\Delta\left(\nu, \nu_{B}, Q^{2}\right)=\delta_{0}+\delta_{\nu} \frac{\nu^{2}}{M_{\pi}^{2}}+\delta_{B} \frac{\nu_{B}}{M_{\pi}}+\delta_{Q} Q^{2} / M_{\pi}^{2}+\ldots
$$

The dispersive amplitude in Eq. (36) is evaluated by the dispersion integral at $t=t_{\mathrm{thr}}\left(Q^{2}\right)$ along the path from $\nu=\nu_{\text {thr }}\left(Q^{2}\right)$ to infinity. In the soft-pion kinematics, the threshold moves to $\nu=0$ and $\nu_{B}=0$ (or $t=M_{\pi}^{2}-Q^{2}$ as long as the pion mass is finite). For small values of $\nu$ and $\nu_{B}$ we can use Eq. (37) to extrapolate from the physical to the soft-pion threshold. Of course, we can not expect to reproduce the FFR sum rule in this way, because the expansion coefficients in Eq. (37) depend on the pion mass and the dispersion calculation only provides these coefficients for the physical mass. In particular the pion loop effects at threshold depend on the pion mass and, moreover, produce a $Q^{2}$ dependence very different from the nucleon form factors. However, from previous experience [1,2] we might expect a suppression of these loop effects if the dispersion integral is evaluated in the sub-threshold region. In Fig. 5 we compare the Pauli form factor $F_{2}^{p}\left(Q^{2}\right)$ (dotted line) to the $Q^{2}$ dependence of $A_{1 \text {, disp }}^{\left(p \pi^{0}\right)}$ as evaluated by the dispersion integral at $\nu=0$ and $\nu=\nu_{\text {thr }}\left(Q^{2}\right)$. Whereas the deviations from the sum rule are quite sizeable at physical threshold (thick lines), they indeed decrease if $\nu$ moves towards 0 . Only slight changes occur if we further extrapolate from $t=t_{\mathrm{thr}}\left(Q^{2}\right)$ to the soft-pion kinematics (thin lines) at $\nu=\nu_{B}=0$. However, Fig. 5 clearly demonstrates that the slopes of the Pauli form factor and the invariant amplitude $A_{1 \text {, disp }}^{\left(p \pi^{0}\right)}$ differ quite a bit. Even the extrapolation of the invariant amplitude to the soft-pion kinematics yields an effective r.m.s. radius much larger than the Pauli radius of the proton, $r_{2}^{p}=0.894 \mathrm{fm}$ of Ref. [27] or $r_{2}^{p}=0.879 \mathrm{fm}$ of Ref. [28]. Let us now study the physics behind these deviations by looking at the integrand and the multipole decomposition of the dispersion integral. The integrand for the amplitude $A_{1 \text {, disp }}^{\left(p \pi^{0}\right)}$ is shown in the top panels of Fig. 6 for the momentum transfers $Q^{2}=0$ and $Q^{2}=0.1 \mathrm{GeV}^{2}$. Evidently the bulk contribution to the integral stems from the $\Delta(1232)$ resonance. In the real photon limit and for energies near threshold (solid line) also the S-wave threshold production is quite 

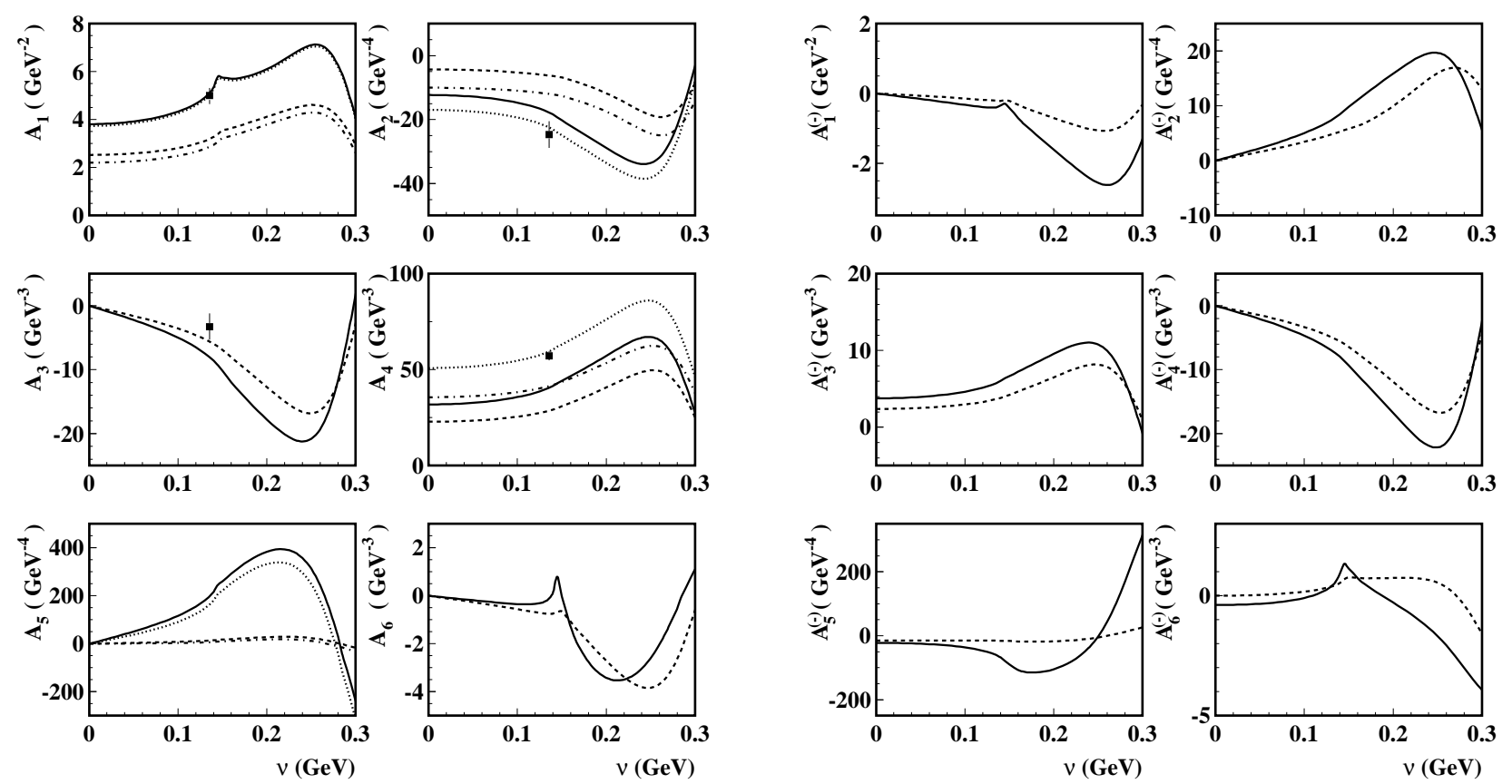

Figure 2. The invariant amplitudes for pion electroproduction at $t=t_{\mathrm{thr}}\left(Q^{2}\right)$ as function of $\nu$. Solid lines: dispersive contributions for $Q^{2}=0$, dashed lines: same at $Q^{2}=0.1 \mathrm{GeV}^{2}$. In the $\mathrm{p} \pi^{0}$ channel (left panels), the inclusion of the vector meson poles leads to the dotted $\left(Q^{2}=0\right)$ and dashed-dotted lines $\left(Q^{2}=0.1 \mathrm{GeV}^{2}\right)$. The data points for real photons are derived from the experimental values of Ref. [26]. The isospin $(-)$ channel is shown in the right panels.

sizeable, but this contribution of the pion cloud decreases rapidly if the energy moves into the sub-threshold region (dashed line). It is also seen that the loop effects drop faster with momentum transfer $Q^{2}$ than the resonance contributions. The contributions of the most important multipoles at $Q^{2}=0$ are, in units of $\mathrm{GeV}^{-2}$,

$$
\begin{aligned}
& A_{1, \operatorname{disp}}^{\left(p \pi^{0}\right) \operatorname{thr}}(0)=1.97\left(E_{0+}\right)+3.59\left(M_{1+}\right)-0.41\left(M_{1-}\right) \\
& \quad+0.10\left(E_{2-}\right)-0.30\left(M_{2-}\right)+0.17 \text { (others) } \\
& \quad=5.12
\end{aligned}
$$

This has to be compared with the sum rule value given by Eq. (24),

$$
A_{1, \mathrm{FFR}}\left(Q^{2}\right)=\left(4.14-13.68 \frac{Q^{2}}{\mathrm{GeV}^{2}}+\ldots\right) \mathrm{GeV}^{-2} .
$$

The difference between the dispersive calculation and the FFR prediction demonstrates the importance of the pion loops near threshold. If the integral is evaluated at $\nu=0$, the S-wave contribution decreases to $1.35 \mathrm{GeV}^{-2}$ and the total result is $A_{1, \text { disp }}^{\left(p \pi^{0}\right)}\left(\nu=0, t_{\mathrm{thr}}, Q^{2}=0\right)=3.81 \mathrm{GeV}^{-2}$. Finally, the extrapolation to the soft-pion kinematics leads to $A_{1 \text {, disp }}^{\left(p \pi^{0}\right)}\left(\nu=\nu_{B}=Q^{2}=0\right)=3.90 \mathrm{GeV}^{-2}$, quite close

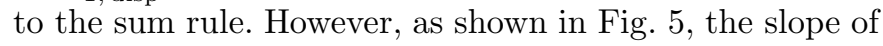
this function differs from the FFR prediction even in the sub-threshold region $(\nu \rightarrow 0)$. The reason for this behavior is already seen in a simple model including the loop contributions in the $\mathrm{S}$ waves, see Eqs. (28) and (29), plus the FFR contributions for all the multipoles (see Appendix E), both in the unexpanded form. Within this model we obtain the following slope for the threshold amplitude and its contributions, all in units of $\mathrm{GeV}^{-4}$ :

$$
\begin{aligned}
& \frac{d}{d Q^{2}} A_{1, \text { disp }}^{\left(p \pi^{0}\right)}(\text { model }) \\
& \quad=-311\left(E_{0+}\right)+278\left(L_{0+}\right)-1(\text { P waves }) \\
& \quad=-13.5(\text { FFR })-20.5(\text { loop })=-34 .
\end{aligned}
$$

The strong cancelation of the transverse and longitudinal $\mathrm{S}$-wave contributions is remarkable. We conclude that a precise knowledge of both multipoles is required in order to get a reliable prediction for the slope. Furthermore, the slope receives large loop contributions. Translated into transition radii, the FFR term has the radius of the Pauli form factor, $r_{2}^{p} \approx 0.88 \mathrm{fm}$, whereas the pion cloud reaches to a much larger distance described by $r$ loop] $\approx$ $1 / M_{\pi}=1.45 \mathrm{fm}$. The total result is $r[$ model $]=1.12 \mathrm{fm}$, in good agreement with the following results obtained from the dispersion integral: $r\left[A_{1, \mathrm{disp}}^{\left(p \pi^{0}\right)}\left(\nu_{\mathrm{thr}}, t_{\mathrm{thr}}, 0\right)\right]=1.16 \mathrm{fm}$, $r\left[A_{1, \text { disp }}^{\left(p \pi^{0}\right)}\left(0, t_{\mathrm{thr}}, 0\right)\right]=1.08 \mathrm{fm}$, and $r\left[A_{1, \mathrm{disp}}^{\left(p \pi^{0}\right)}\left(0, M_{\pi}^{2}, 0\right)\right]=$ $1.13 \mathrm{fm}$. In conclusion, the FFR sum rule can not be used to determine the Pauli form factor from the $Q^{2}$ dependence of the invariant amplitude $A_{1}$. The radius derived from that observable is about $25 \%$ larger than the Pauli radius, which is another "smoking gun" for the importance of the pion cloud in low-energy nuclear physics. 

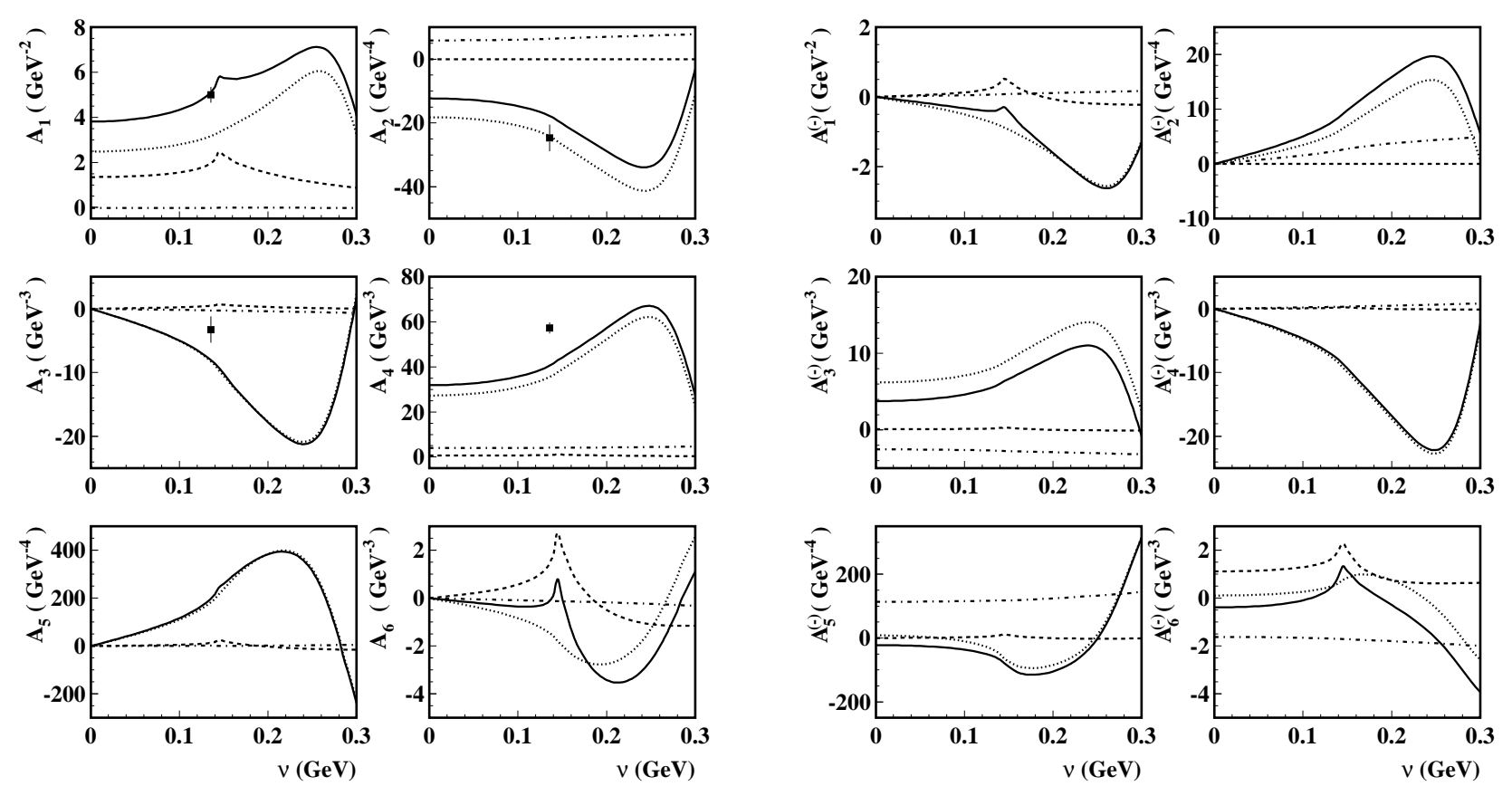

Figure 3. Contributions to the invariant amplitudes from the imaginary part of $\mathrm{S}$ waves (dashed lines), $\mathrm{P}$ waves (dotted lines), D plus F waves (dashed-dotted lines), and total result (solid lines). See Fig. 2 for further notation.

\subsection{The FFR sum rule for the invariant amplitudes $A_{6}$}

Let us now turn to Eq. (25), which connects the axial and Dirac isovector form factors with the amplitude $A_{6}^{(-)}\left(\nu, t_{\mathrm{thr}}\left(Q^{2}\right), Q^{2}\right)$. The isovector Dirac radius is relatively well known from various analyses of elastic electron scattering, e.g., $\left\langle r^{2}\right\rangle_{1}^{V}=(0.585 \pm 0.010) \mathrm{fm}^{2}$ [27]. The axial mass parameter as determined by neutrino and antineutrino scattering [29] lies in the range of $M_{A}=$ $(1.026 \pm 0.021) \mathrm{GeV}$ corresponding to $\left\langle r^{2}\right\rangle_{A}^{V}=(0.444 \pm$ $0.019) \mathrm{fm}^{2}$ 30. With these values we obtain

$$
A_{6, \mathrm{FFR}}(0)=(1.31 \pm 0.27) \mathrm{GeV}^{-3},
$$

and with the same axial mass but the form factor parametrization of Kelly [28]

$$
A_{6, \mathrm{FFR}}\left(Q^{2}\right)=\left(1.54-9.48 \frac{Q^{2}}{\mathrm{GeV}^{2}}+\ldots\right) \mathrm{GeV}^{-3} .
$$

According to Eq. (64) of Appendix [C only the S-wave multipole $E_{0+}^{(-)}\left(Q^{2}\right)$ survives in the soft-pion limit $\mu \rightarrow 0$ as long as $Q^{2}$ is finite. In accordance with the LET of Nambu et al. 21, the information of the LET resides in the slope of that multipole. On the other hand, the FFR current is purely longitudinal for $Q^{2}=0$ and finite pion mass (see Appendix E). For the form factor parametrization of Kelly [28, the multipole $L_{0+}^{(-)}$accounts for $94 \%$ of $A_{6, \mathrm{FFR}}$ at $Q^{2}=0$, the remainder being given by $L_{1-}^{(-)}$. Already at $Q^{2}=0.1 \mathrm{GeV}^{2}$, the bulk contribution (78\%) is due to the rising multipole $E_{0+}^{(-)}$. In the real world of finite pion masses, the situation is more complicated. The integrand for the amplitude $A_{6, \text { disp }}^{(-)}$is shown in the lower panels of Fig. 6 for the momentum transfers $Q^{2}=0$ and $Q^{2}=0.1 \mathrm{GeV}^{2}$. The figure shows positive contributions from both threshold pion production and $\Delta(1232)$ resonance excitation. However, these contributions are largely canceled by equally strong ones with opposite signs in the second and third resonance regions. In order to quantify this effect, we decompose the imaginary part of the amplitude into a multipole series. In this way the dispersive photoproduction amplitude at the cusp takes the following form:

$$
\begin{aligned}
& A_{6, \text { disp }}^{(-) \text {cusp }}(0)=3.82\left(E_{0+}\right)-1.49\left(L_{0+}\right)+0.91\left(M_{1+}\right) \\
& +1.05\left(E_{1+}\right)-0.95\left(L_{1+}\right)+0.05\left(M_{1-}\right)-0.38\left(L_{1+}\right) \\
& -1.80\left(E_{2-}\right)+0.06\left(M_{2-}\right)+0.38\left(L_{2-}\right)-0.33 \text { (rest) } \\
& =2.33(\mathrm{~S})+0.68(\mathrm{P})-1.34(\mathrm{D})-0.35(\mathrm{~F})=1.32,(43)
\end{aligned}
$$

with all the values given in units of $\mathrm{GeV}^{-3}$. The dispersive amplitude at the cusp, Eq. (43), confirms the FFR sum rule value given by Eq. (41). This is surprising because of the formidable cancelations occurring in Eq. (43). We observe a substantial cancelation both among multipoles with the same pion partial wave and between the strong electromagnetic dipole excitations $E_{0+}^{(-)}$and $E_{2-}^{(-)}$. It is also remarkable that the electric transverse and longitudinal multipoles of the $\Delta(1232)$ resonance, $E_{1+}^{(-)}$and $L_{1+}^{(-)}$, contribute just as much as the magnetic $M_{1+}^{(-)}$ transition, although the latter multipole is stronger by factors of 40 and 25, respectively. Furthermore, both $\mathrm{S}$ and $\mathrm{P}$ waves yield positive contributions, whereas the $\mathrm{D}$ and $\mathrm{F}$ waves of the second and third resonance regions diminish the integral. As $\nu$ moves from the cusp 

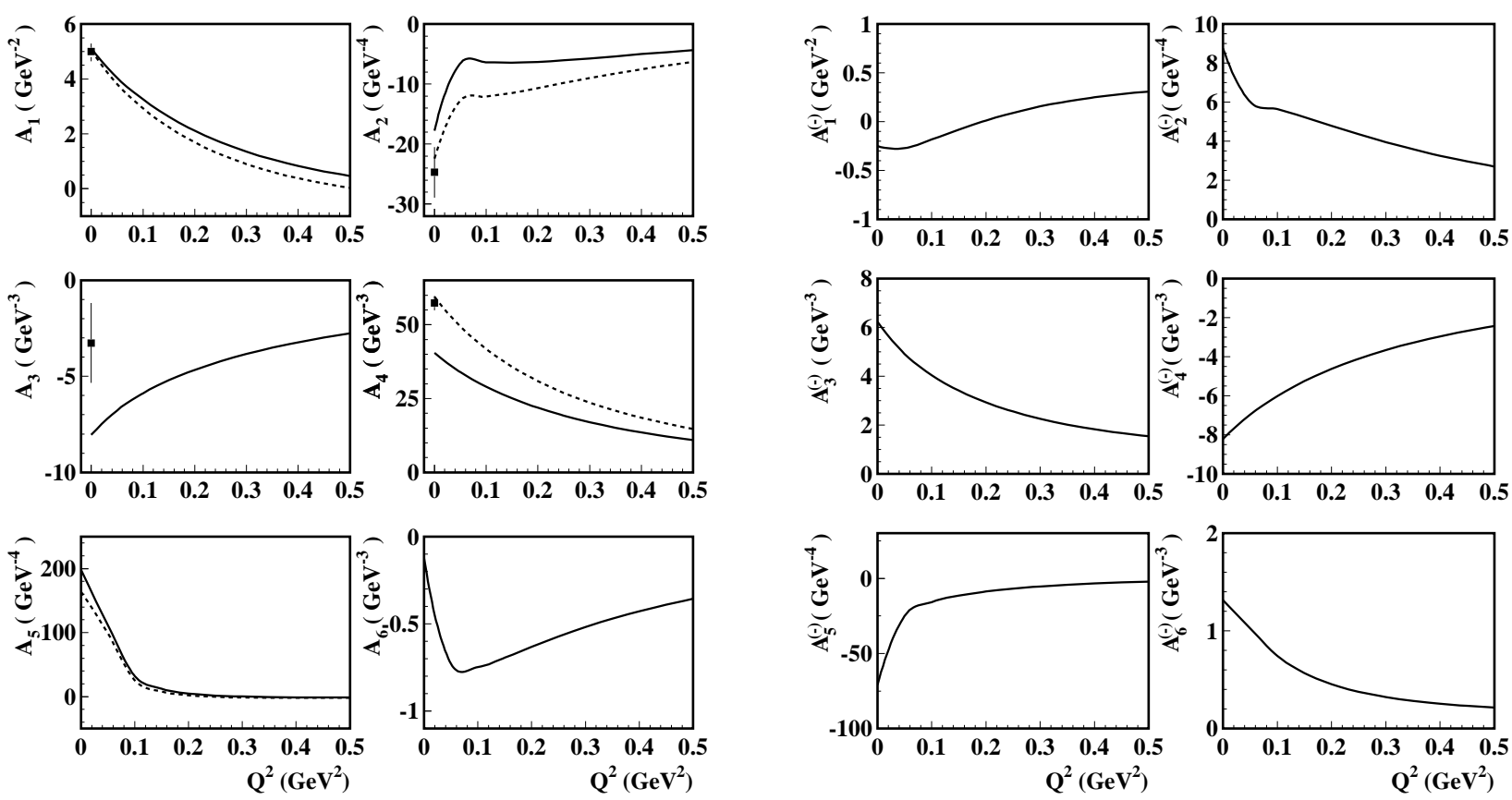

Figure 4. The invariant amplitudes for pion electroproduction at $t=t_{\mathrm{thr}}\left(Q^{2}\right)$ and $\nu=\nu_{\mathrm{thr}}\left(Q^{2}\right)$ as function of $Q^{2}$. For the $p \pi^{0}$ channel (left panel) the inclusion of the vector meson poles leads to the dashed curves.
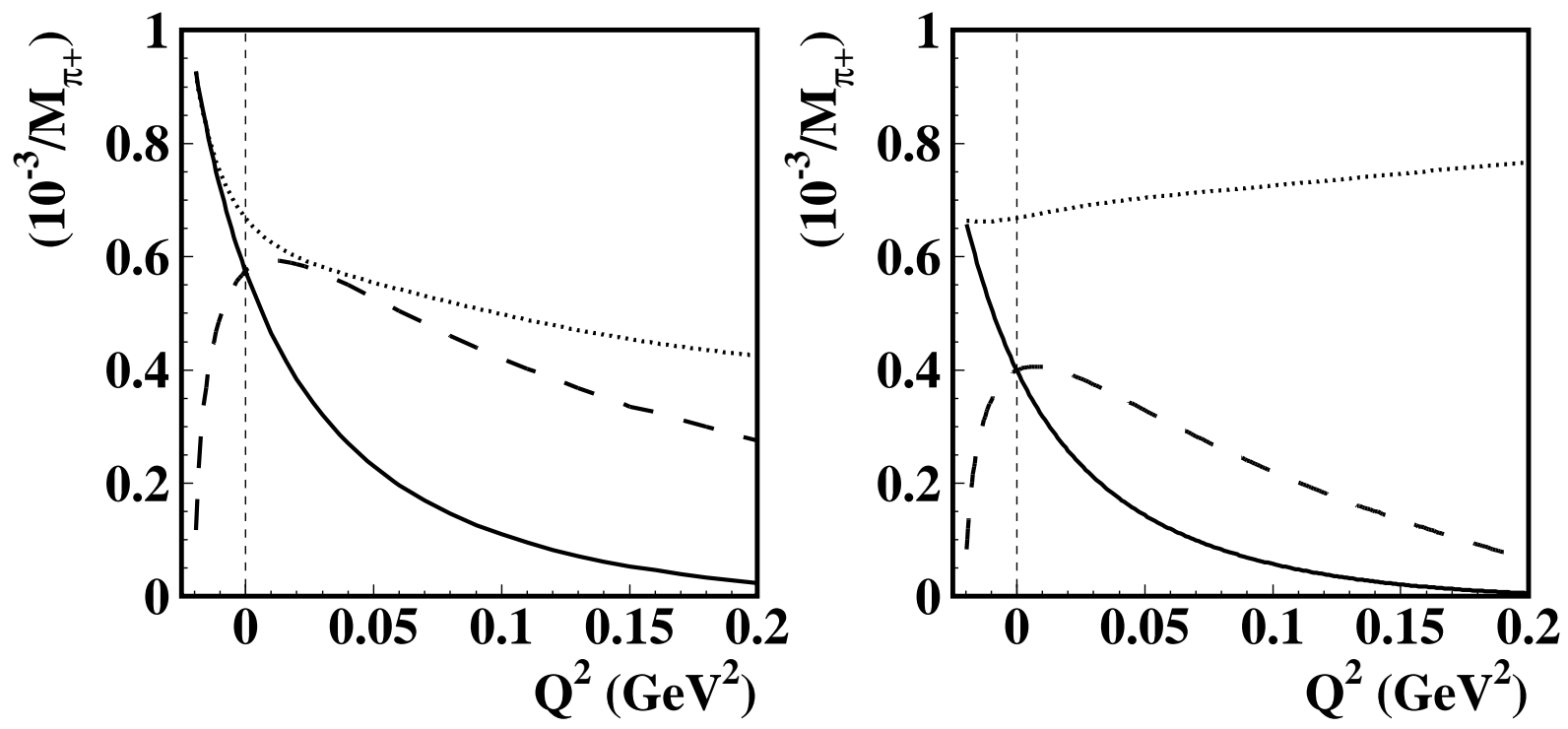

Figure 7. The S-wave multipoles $E_{0+}^{(-)}$(dotted lines), $L_{0+}^{(-)}$(solid lines), and $S_{0+}^{(-)}$(dashed lines) as function of $Q^{2}$ at $\nu=\nu_{\mathrm{thr}}\left(Q^{2}\right)$ and $t=t_{\mathrm{thr}}\left(Q^{2}\right)$, in units of $10^{-3} / M_{\pi^{+}}$. Left panel: results with MAID05 as input for the dispersion integral, right panel: same with MAID07 [31. Since the dispersive contributions are only a small fraction of the full multipoles, the shown deviations between the two version of MAID are within the present experimental error bars.

value to $\nu=0$, the total $\mathrm{S}$-wave contribution decreases from $2.33 \mathrm{GeV}^{-3}$ to $1.12 \mathrm{GeV}^{-3}$ and the $\Delta(1232)$ contribution drops from $1.01 \mathrm{GeV}^{-3}$ to $0.59 \mathrm{GeV}^{-3}$, whereas the higher multipole contributions change little. As a result the invariant amplitude becomes negative, $A_{6, \text { disp }}^{(-)}\left(\nu=0, t_{\mathrm{thr}}, 0\right)=-0.38 \mathrm{GeV}^{-3}$. Incidentally, the discussed model of loop and FFR terms yields a contribution of $4.05 \mathrm{GeV}^{-3}$ from the real part of the threshold multipole $E_{0+}^{(-)}$, in qualitative agreement with Eq. (43). However, the multipole contributions of Eq. (43) are defined by a decomposition of the imaginary part, and a particular multipole in the imaginary part will generally contribute to all the multipoles in the real part. Furthermore, the large cancelation due to higher resonances would have to be described by appropriate 


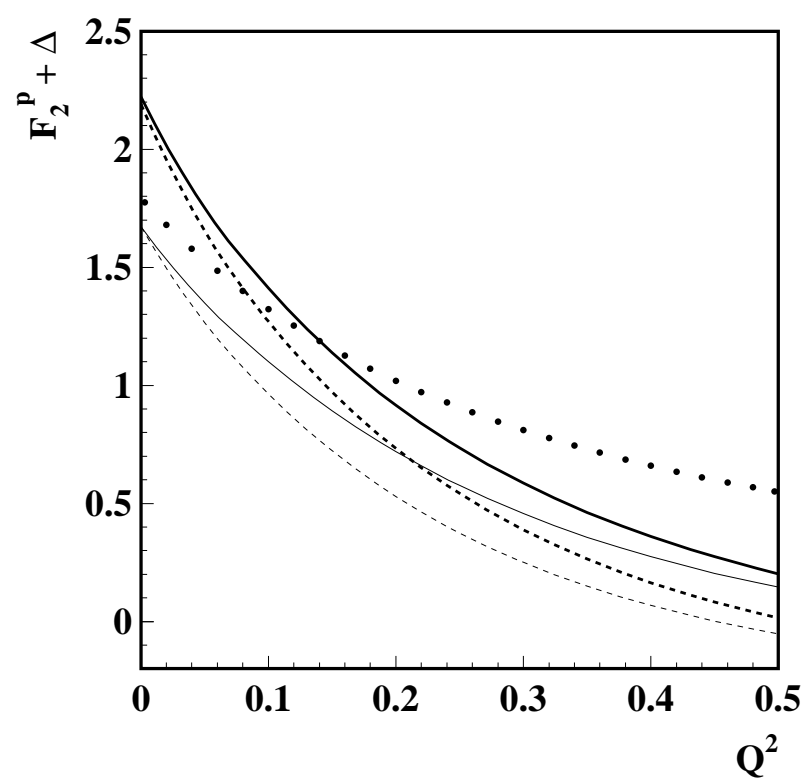

Figure 5. The proton's Pauli form factor $F_{2}^{p}$ as function of $Q^{2}$, compared to the result of dispersion theory as obtained from the rhs of Eq. (1). Thick solid line: dispersive results for the threshold amplitude $\left(\nu=\nu_{\mathrm{thr}}\left(Q^{2}\right), t=t_{\mathrm{thr}}\left(Q^{2}\right)\right)$, thick dashed line: same kinematics but including the $t$-channel vector meson poles, thin solid line: dispersive results for soft-pion kinematics $\left(\nu=\nu_{B}=0\right)$, thin dashed line: same kinematics but including the $t$-channel vector meson poles. The dashed-dotted line is the parametrization of $F_{2}^{p}$ according to Ref. [28].

low-energy constants in an effective field theory.

In spite of the modern precision data serving as input for the dispersive calculation, the results still keep changing. This is demonstrated by Fig. 7 showing the S-wave multipoles at threshold as function of $Q^{2}$. The left panel displays these multipoles with input from MAID05, the right panel is obtained with the recent version MAID07 31 containing many new charged-pion data from recent JLab experiments. Figure 7 shows several constraints necessary for a meaningful description of the threshold data: (I) In the Siegert limit, $|\mathbf{k}| \rightarrow 0$ or $Q^{2} \rightarrow-M_{\pi^{+}}^{2}$, the transverse electric multipole $E_{0+}^{(-)}$coincides with the longitudinal multipole $L_{0+}^{(-)}$. (II) Gauge invariance requires that $|\mathbf{k}| L_{0+}^{(-)}=k_{0} S_{0+}^{(-)}$, and therefore the scalar or Coulomb multipole $S_{0+}^{(-)}$must be equal to the longitudinal multipole $L_{0+}^{(-)}$at the real photon point, $Q^{2}=0$. (III) Because $k_{0}$ vanishes for $Q^{2}=2 M_{N} M_{\pi^{+}}+$ $M_{\pi^{+}}^{2}=0.28 \mathrm{GeV}^{2}$, gauge invariance also implies that the longitudinal multipole $L_{0+}^{(-)}$decreases with $Q^{2}$ towards a zero at this point. The comparison between the results based on MAID05 and MAID07 shows that $E_{0+}^{(-)}$has not changed much, except for a change of the slope from negative to positive, the latter being in qualitative agreement with Eq. (30) although at a considerably smaller value. However, the larger data base of MAID07 leads to a much
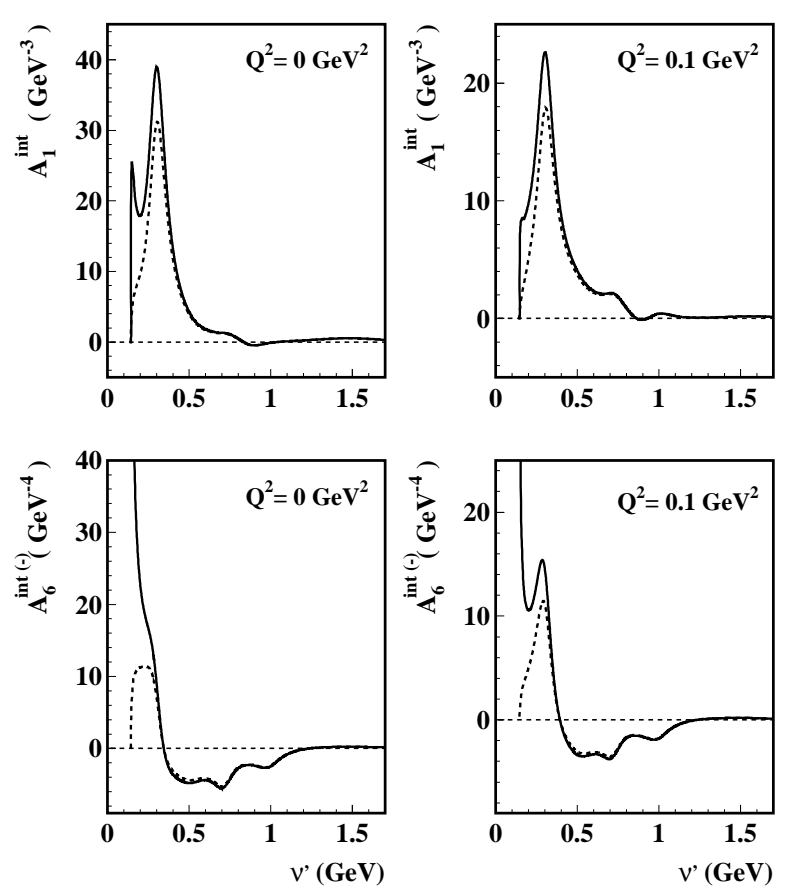

Figure 6. The integrands of the dispersion integrals for $A_{1}^{\left(p \pi^{0}\right)}$ and $A_{6}^{(-)}$are shown in the upper and lower rows, respectively. Left: $Q^{2}=0$, right: $Q^{2}=0.1 \mathrm{GeV}^{2}$; solid lines: integrands obtained for $\nu=\nu_{\mathrm{thr}}\left(Q^{2}\right)$, dashed lines: integrands obtained for $\nu=0$.

smaller value for the longitudinal multipole $L_{0+}^{(-)}$, which is now at half-way between MAID05 and the tiny value given by Eq. (31). These large differences for the dispersive amplitudes are, however, small in view of the huge pole terms. A comparison of Fig. 7 with the pole term contributions of Eqs. (30) and (31) shows that the seemingly large changes of the dispersive contributions amount at most to a few per cent of the total amplitude. However, since the FFR sum rule is based on the dispersive contributions, the agreement of Eq. (43) with the sum rule must be taken with a grain of salt.

\subsection{The pion radius and the invariant amplitude $A_{5}$}

As pointed out by Bernard et al. [19], the slope of $L_{0+}^{(-)}$, disp given by Eq. (31),

$$
\frac{d}{d Q^{2}} L_{0+, \text { disp }}^{(-) \operatorname{thr}} \approx-13.7 \cdot \frac{10^{-3}}{\mathrm{GeV}^{2} M_{\pi^{+}}}
$$

is comparable with the contribution of the pion radius to the slope of the pole term, see Eq. (68) of Appendix D

$$
\frac{d}{d Q^{2}} L_{0+, \pi \text { pole }}^{(-) \text {thr }} \approx-22.8 \cdot \frac{10^{-3}}{\mathrm{GeV}^{2} M_{\pi^{+}}} .
$$

Therefore, the dispersive contribution to the slope is expected to simulate a substantial increase of the pion radius 

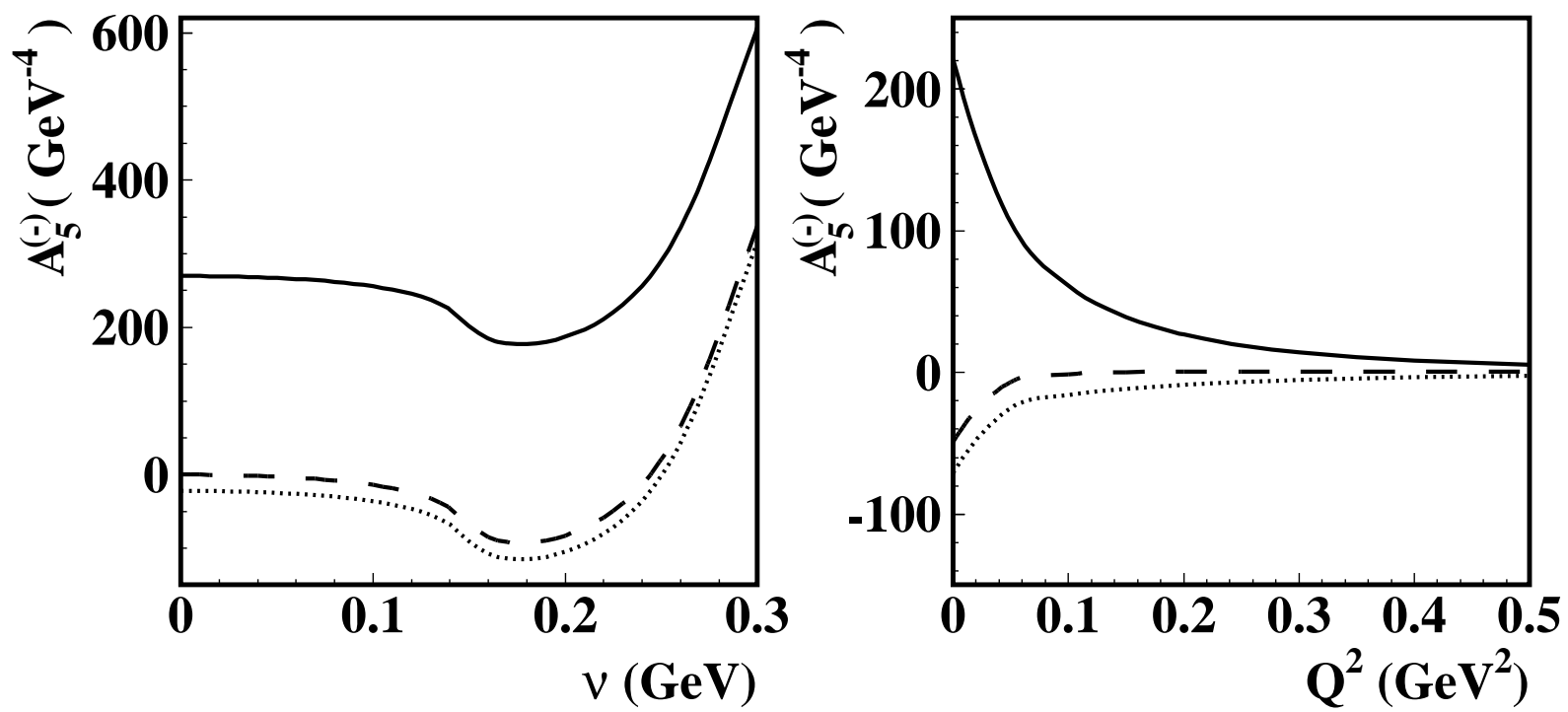

Figure 8. The invariant amplitude $A_{5, \text { disp }}^{(-)}$obtained from the unsubtracted DR Eq. (12) (solid lines), the subtracted DR of Eq. (48) (dashed lines), and Eq. (22) as proposed by von Gehlen (dotted lines), in units of $\mathrm{GeV}^{-4}$. Left panel: $A_{5, \mathrm{disp}}^{(-)}$as function of the crossing-symmetric variable $\nu$ and at fixed values $t=t_{\mathrm{thr}}(0)$ and $Q^{2}=0$, right panel: $A_{5, \text { disp }}^{(-)}$as function of the virtuality $Q^{2}$ at $\nu=\nu_{\text {thr }}\left(Q^{2}\right)$ and $t=t_{\text {thr }}\left(Q^{2}\right)$.

if the experimental value of $L_{0+}^{(-)}$is simply compared to the pole term. In fact such an analysis yields a modified square radius,

$$
\left\langle\tilde{r}^{2}\right\rangle_{\pi}^{V}=\left\langle r^{2}\right\rangle_{\pi}^{V}+\frac{3}{32 F_{\pi}^{2}}\left(\frac{16}{\pi^{2}}-1\right)
$$

With $\left\langle r^{2}\right\rangle_{\pi}^{V}=0.44 \mathrm{fm}^{2}$ as determined by scattering highenergetic pions off atomic electrons [32 and the loop correction of $0.26 \mathrm{fm}^{2}$ (second term on the rhs of Eq. (46) ), the "effective" value is $\left\langle\tilde{r}^{2}\right\rangle_{\pi}^{V}=0.70 \mathrm{fm}^{2}$, corresponding to an effective pion radius of $0.84 \mathrm{fm}$. Our dispersive analysis confirms these results, although the slope of $L_{0+, \text { disp }}^{(-) \text {thr }}$ differs somewhat from Eq. (44):

$$
\frac{d}{d Q^{2}} L_{0+, \operatorname{disp}}^{(-)}=\left\{\begin{array}{c}
-12.6 \cdot \frac{10^{-3}}{\mathrm{GeV}^{2} M_{\pi^{+}}} \text {[MAID05] } \\
-9.4 \cdot \frac{10^{-3}}{\mathrm{GeV}^{2} M_{\pi^{+}}} \text {[MAID07] }
\end{array}\right.
$$

As has been discussed before, the calculation of the amplitude $A_{5}^{(-)}$, disp requires some care, because the unsubtracted dispersion integral yields a contribution with the shape of the pion pole term. Since the experimental pion form factor is already fully included in the pole term, any additional pole structure at $\nu=0$ and $t=M_{\pi^{+}}^{2}$ ought to be removed from $A_{5 \text {, disp }}^{(-)}$according to Eq. (22). Figure 8 shows that the unsubtracted dispersion integral (solid line) changes dramatically by removing this pole contribution according to von Gehlen [6], see the dotted line. In fact, the integrands for the 2 procedures show a completely different behavior as function of the excitation energy, not only in size but also in sign. Moreover, the unsubtracted dispersion integral does not well converge for $\nu^{\prime} \rightarrow \infty$, see also the work of Manweiler and Schmidt 33 and Aznauryan 34. We have therefore corroborated the procedure of von Gehlen by subtracting the DR at $\nu=0$ and at the given $t_{\mathrm{thr}}\left(Q^{2}\right)$,

$$
\begin{aligned}
& A_{5, \operatorname{disp}}^{(-)}\left(\nu, t, Q^{2}\right)=A_{5, \operatorname{disp}}^{(-)}\left(0, t, Q^{2}\right) \\
& \quad+\frac{2 \nu^{2}}{\pi} \mathcal{P} \int_{\nu_{t h r}}^{\infty} \mathrm{d} \nu^{\prime} \frac{\operatorname{Im} A_{i}^{(I)}\left(\nu^{\prime}, t, Q^{2}\right)}{\nu^{\prime}\left(\nu^{\prime 2}-\nu^{2}\right)} .
\end{aligned}
$$

The results are shown by the dashed lines in Fig. 8, They differ from the dotted lines by an energy-independent shift, $\left.A_{5}^{(-)}\left(Q^{2}\right)\right|_{\mathrm{vG}} \equiv A_{5, \operatorname{disp}}^{(-)}\left(\nu=0, t_{\mathrm{thr}}\left(Q^{2}\right), Q^{2}\right)$, as obtained from von Gehlen's procedure. In view of the excellent convergence of the subtracted DR, Eq. (48), we rather prefer this equation for further studies. In principle, the subtraction function $A_{5 \text {,disp }}^{(-)}\left(0, t, Q^{2}\right)$ can be fitted to the data by adding a polynomial in $t-M_{\pi}^{2}$ and $Q^{2}$, or by a dispersive approach in the variable $t$. However, we have chosen $\left.A_{5}^{(-)}\left(Q^{2}\right)\right|_{\mathrm{vG}}$ for the present discussion of the lowenergy region. The near perfect agreement with the subtracted DR shows that the subtraction function has only a negligible dependence on $t$ but a rapid decrease with $Q^{2}$, at least in the threshold region.

\section{Summary and Discussion}

We have studied the relativistic amplitudes for pion electroproduction on the nucleon in the framework of dispersion relations at constant $t$. This procedure allows us to determine these amplitudes not only in the physical region but also for sub-threshold energies. In the latter 
region, the dispersive amplitudes are regular functions which can be expanded as a (real) power series in the independent kinematic variables. A comparison of this series with the results of ChPT yields the low-energy constants of that theory through global properties of the excitation spectrum.

The present work has concentrated on the threshold region in order to compare our findings with the predictions of several low-energy theorems based on threshold production in the soft-pion limit. In general agreement with ChPT, we find large corrections to these theorems due to the finite pion mass. In particular we have studied two sum rules of Fubini et al., which connect (I) the transverse amplitude $A_{1}^{\left(N \pi^{0}\right)}$ for neutral pion production with the Pauli form factor $F_{2}^{N}\left(Q^{2}\right)$ of the nucleon and (II) the longitudinal amplitude $A_{6}^{(-)}$for charged pion production with the nucleon's axial form factor. As was shown in previous work on neutral pion photoproduction, the former sum rule describes the anomalous magnetic moment $\kappa_{N}=F_{2}^{N}(0)$ in the subthreshold region to an accuracy of a few per cent, whereas there are sizeable unitary (rescattering) corrections at the physical threshold. The extension to electroproduction yields large corrections both at and below threshold, which can be visualized by pion-loop contributions occurring at a radius of about $1.4 \mathrm{fm}$ and therefore leading to a total transition radius distinctly larger than the Pauli radius. The second sum rule is based on the facts that (I) the pole contribution to $A_{6}^{(-)}$vanishes and (II) chiral invariance leads to the Kroll-Ruderman term as leading threshold contribution, with the consequence that the isovector Dirac form factor has to be replaced by the axial form factor. As a result the dispersive amplitude for the isospin (-) amplitude is proportional to $\left\langle r^{2}\right\rangle_{1}^{V}-\left\langle r^{2}\right\rangle_{A}^{V}$ in the soft-pion limit. The dispersive approach reproduces this sum rule at threshold, albeit at the expense of terrific cancelations among the photoproduction multipoles stemming from different parts of the excitation spectrum. However, the dispersive amplitude changes rapidly if we move away from threshold, the onset of the imaginary part, and also as function of $Q^{2}$.

We have furthermore studied the second longitudinal amplitude $A_{5}^{(-)}$, which is known to converge badly. Indeed we have found that the unsubtracted dispersion integral leads to a huge contribution with the structure of the pion pole term, and as a consequence to unrealistic multipoles, particularly for the longitudinal ones. If we subtract this contribution, the threshold amplitude changes from large positive to small negative values. In order to corroborate this result we have also set up a subtracted dispersion relation for $A_{5}^{(-)}$. The latter procedure is in perfect agreement with the described recipe to eliminate the pion pole structure, except for the off-set at the subtraction point. In agreement with ChPT, our analysis yields a dispersive contribution to the longitudinal S-wave multipole $L_{0+}^{(-)}$with a large negative slope in $Q^{2}$, which is comparable to the slope of the pole contribution. If the experimental value of $L_{0+}^{(-)}$is simply compared to the pion pole term, this dispersive effect simulates an increase of the pion radius by about $20-25 \%$.

In conclusion, dispersion relations allow us to construct a unitary, gauge and Lorentz invariant description of pion electroproduction on the nucleon. They are based on the available experimental information for the absorptive parts of the multipoles as contained, for example, in the MAID or SAID 35 data analysis. In this work we have concentrated on the threshold amplitudes in order to compare with sum rules and the predictions of ChPT. In a next step we plan to extend our calculations to higher energies up to the $\Delta(1232)$ resonance in order to constrain the real parts of the background multipoles, which still prevent us from a truly model-independent determination of the small electric and Coulomb amplitudes for $\Delta(1232)$ excitation. Also along the same lines, it is our aim to tackle the still existing discrepancies between the electroproduction data for neutral pion production near threshold and the respective theoretical predictions, from both ChPT and phenomenological models like MAID. We hope that this future work will provide many new cross-checks with relativistic effective field theories, improve our knowledge of pion electroproduction as a means to get insight into the spatial structure of this reaction, and lead to a better understanding of the interplay between pion-cloud and resonance effects in the nucleon.

\section{Acknowledgements}

This work was supported by the Deutsche Forschungsgemeinschaft (SFB 443) and the EU Integrated Infrastructure Initiative Hadron Physics Project under contract number RII3-CT-2004-506078.

\section{References}

1. B. Pasquini, D. Drechsel, and L. Tiator, Eur. Phys. J. A 23 (2005) 279.

2. B. Pasquini, D. Drechsel, and L. Tiator, Eur. Phys. J. A 27 (2006) 231.

3. P. Dennery, Phys. Rev. 124 (1961) 2000.

4. J.S. Ball, Phys. Rev. 124 (1961) 2014.

5. F.A. Berends, A. Donnachie, and D.L. Weaver, Nucl. Phys. B 4 (1967) 1.

6. G. v. Gehlen, Nucl. Phys. B9 (1969) 17.

7. V. Bernard, N. Kaiser, and U.-G. Meißner, Nucl. Phys. B 383 (1992) 442.

8. V. Bernard, N. Kaiser, T-S.H. Lee, and U.-G. Meißner, Physics Reports 246 (1994) 315.

9. V. Bernard, L. Elouadrhiri, and U.-G. Meißner, J. Phys. G 28 (2002) R1.

10. V. Bernard, B. Kubis, and U.-G. Meißner, Eur. Phys. J. A 25 (2005) 419.

11. B. Lehnhart, PhD thesis, Mainz 2007, http://archimed.uni-mainz.de/ B. Lehnhart et al., in preparation. 
12. D. Drechsel, O. Hanstein, S.S. Kamalov, and L. Tiator, Nucl. Phys. A 645 (1999) 145; http://www.kph.uni-mainz.de/MAID/

13. N.M. Kroll and M.A. Ruderman, Phys. Rev. 93 (1954) 233.

14. A.I. Vainshtein and V.I. Zakharov, Yad. Fiz. 12 (1970) 610 [Sov. J. Nucl. Phys. 12 (1970) 333].

15. P. de Baenst, Nucl. Phys. B 24 (1970) 633.

16. Bernard, N. Kaiser, J. Gasser, and U.-G. Meißner, Phys. Lett. B 268 (1991) 291.

17. V. Bernard, N. Kaiser, and U.-G. Meißner, Phys. Lett. B 282 (1992) 448.

18. V. Bernard, N. Kaiser, and U.-G. Meißner, Phys. Rev. Lett. 69 (1992) 1877.

19. V. Bernard, N. Kaiser, and U.-G. Meißner, Phys. Rev. C 62 (2000) 028201.

20. S. Fubini, G. Furlan, and C. Rossetti, Nuovo Cimento 40 (1965) 1171.

21. Y. Nambu and D. Lurié, Phys. Rev. 125 (1962) 1429; Y. Nambu and E. Shrauner, Phys. Rev. 128 (1962) 862; Y. Nambu and M. Yoshimura, Phys. Rev. Lett. 24 (1970) 25 .

22. Riazuddin and B.W. Lee, Phys. Rev. 146 (1966) 1202.

23. S.L. Adler and F.J. Gilman, Phys. Rev. 152 (1966) 1460.

24. J.D. Bjorken and S.D. Drell, "Relativistic quantum fields", (McGraw-Hill, New York, 1965).

25. G.F. Chew et al., Phys. Rev. 106 (1957) 1345.

26. A. Schmidt et al., Phys. Rev. Lett. 87 (2001) 232501.

27. P. Mergell, U.-G. Meißner, and D. Drechsel, Nucl. Phys. A 596 (1996) 367.

28. J.J. Kelly, Phys. Rev. C 70 (2004) 068202.

29. L.A. Ahrens et al., Phys. Lett. B 202 (1988) 284.

30. A. Liesenfeld et al. (A1 Collaboration), Phys. Lett. B 468 (1999) 20.

31. D. Drechsel, S.S. Kamalov, and L.Tiator, Eur. Phys. J. A34 (2007) 69.

32. S.R. Amendolia et al. (NA 7 Collaboration), Nucl. Phys. B 277 (1986) 168.

33. R.W. Manweiler and W. Schmidt, Phys. Rev. D 3 (1971) 2752 .

34. I.G. Aznauryan, Phys. Rev. C 67 (2003) 015209.

35. R.A. Arndt, W.J. Briscoe, I.I. Strakovsky, and R.L. Workman, Phys. Rev. C 66 (2002) 055213; http://gwdac.phys.gwu.edu/ 


\section{Appendix}

\section{A - Expansion of invariant amplitudes in terms of CGLN amplitudes}

Introducing the combinations

$$
\mathcal{X}_{m n}^{( \pm)}=\frac{1}{E_{i}-M_{N}} \mathcal{F}_{m} \pm \frac{E_{f}+M_{N}}{k q} \mathcal{F}_{n}, \mathcal{Y}_{m n}^{( \pm)}=\frac{W-M_{N}}{E_{i}-M_{N}} \mathcal{F}_{m} \pm \frac{\left(W+M_{N}\right)\left(E_{f}+M_{N}\right)}{k q} \mathcal{F}_{n}
$$

we can cast these relations into the form

$$
\begin{aligned}
& \mathcal{N} A_{1}=\left(W^{2}-M_{N}^{2}+Q^{2}\right) \mathcal{X}_{12}^{(-)}+M_{N} \frac{k_{0}\left(t-M_{\pi}^{2}+Q^{2}\right)-2 q_{0} Q^{2}}{k q} \mathcal{X}_{34}^{(+)}+\frac{2 M_{N} Q^{2}}{k_{0}} \mathcal{X}_{56}^{(+)}, \\
& \mathcal{N} A_{2}=-\frac{2 Q^{2}}{\left(t-M_{\pi}^{2}\right)} \mathcal{X}_{12}^{(-)}+\frac{k_{0}\left(t-M_{\pi}^{2}+Q^{2}\right)-2 q_{0} Q^{2}}{k q\left(t-M_{\pi}^{2}\right)} \mathcal{Y}_{34}^{(-)}+\frac{2 Q^{2}}{k_{0}\left(t-M_{\pi}^{2}\right)} \mathcal{Y}_{56}^{(-)}, \\
& \mathcal{N} A_{3}=\mathcal{Y}_{12}^{(+)}+\frac{k_{0}\left(t-M_{\pi}^{2}+Q^{2}\right)-2 q_{0} Q^{2}+4 k^{2} W}{2 k q} \mathcal{X}_{34}^{(+)}+\frac{Q^{2}}{k_{0}} \mathcal{X}_{56}^{(+)} \\
& \mathcal{N} A_{4}=\mathcal{Y}_{12}^{(+)}+\frac{k_{0}\left(t-M_{\pi}^{2}+Q^{2}\right)-2 q_{0} Q^{2}}{2 k q} \mathcal{X}_{34}^{(+)}+\frac{Q^{2}}{k_{0}} \mathcal{X}_{56}^{(+)}, \\
& \mathcal{N} A_{5}=\frac{t-M_{\pi}^{2}-4 M_{N} \nu}{t-M_{\pi}^{2}}\left(\mathcal{X}_{12}^{(-)}-\frac{1}{k_{0}} \mathcal{Y}_{56}^{(-)}\right)+\frac{\left(t-M_{\pi}^{2}\right)\left(k_{0}+q_{0}-2 W\right)+2 M_{N} \nu\left(k_{0}-2 q_{0}\right)}{k q\left(t-M_{\pi}^{2}\right)} \mathcal{Y}_{34}^{(-)} \\
& \mathcal{N} A_{6}=\mathcal{Y}_{12}^{(+)}+2 M_{N} \mathcal{X}_{12}^{(-)}+\frac{\left(t-M_{\pi}^{2}+Q^{2}\right)\left(2 W-k_{0}\right)+2\left(W^{2}-M_{N}^{2}\right) q_{0}}{2 k q} \mathcal{X}_{34}^{(+)}-\frac{W^{2}-M_{N}^{2}}{k_{0}} \mathcal{X}_{56}^{(+)},
\end{aligned}
$$

where $\mathcal{N}=W \sqrt{\left(E_{i}+M_{N}\right)\left(E_{f}+M_{N}\right)} / 2 \pi$.

\section{B - Multipole expansion of CGLN amplitudes}

The multipole series of the CGLN amplitudes takes the form:

$$
\begin{aligned}
\mathcal{F}_{1}= & \sum_{l=0}^{\infty}\left[\left(l M_{l+}+E_{l+}\right) P_{l+1}^{\prime}(x)+\left((l+1) M_{l-}+E_{l-}\right) P_{l-1}^{\prime}(x)\right], \\
\mathcal{F}_{2}= & \sum_{l=1}^{\infty}\left[(l+1) M_{l+}+l M_{l-}\right] P_{l}^{\prime}(x), \\
\mathcal{F}_{3}= & \sum_{l=1}^{\infty}\left[\left(E_{l+}-M_{l+}\right) P_{l+1}^{\prime \prime}(x)+\left(E_{l-}+M_{l-}\right) P_{l-1}^{\prime \prime}(x)\right], \\
\mathcal{F}_{4}= & \sum_{l=2}^{\infty}\left[M_{l+}-E_{l+}-M_{l-}-E_{l-}\right] P_{l}^{\prime \prime}(x), \\
\mathcal{F}_{5}= & \sum_{l=0}^{\infty}\left[(l+1) L_{l+} P_{l+1}^{\prime}(x)-l L_{l-} P_{l-1}^{\prime}(x)\right], \\
\mathcal{F}_{6}= & \sum_{l=1}^{\infty}\left[l L_{l-}-(l+1) L_{l+}\right] P_{l}^{\prime}(x), \\
\mathcal{F}_{7}= & \sum_{l=1}^{\infty}\left[l S_{l-}-(l+1) S_{l+}\right] P_{l}^{\prime}(x), \\
\mathcal{F}_{8}= & \sum_{l=0}^{\infty}\left[(l+1) S_{l+} P_{l+1}{ }^{\prime}(x)-l S_{l-} P_{l-1}^{\prime}(x)\right],
\end{aligned}
$$

where $x=\left(t-M_{\pi}^{2}+Q^{2}+2 q_{0} k_{0}\right) /(2 q k)$ is the cosine of the scattering angle in the physical region. The longitudinal $\left(L_{l}\right)$ and charge $\left(S_{l}\right)$ multipoles are related by gauge invariance, $k_{0} \mathcal{F}_{7}=k \mathcal{F}_{6}$ and $k_{0} \mathcal{F}_{8}=k \mathcal{F}_{5}$. In the limits of $q \rightarrow 0$ (physical threshold) and $k \rightarrow 0$ (pseudothreshold or Siegert limit), the multipoles have the following behavior: $E_{l+}, M_{l+}, L_{l+}, M_{l-} \rightarrow k^{l} q^{l}$ and $E_{l-}, L_{l-} \rightarrow k^{l-2} q^{l}$, with the exception that $L_{1-} \rightarrow k q$. 


\section{C - Multipole expansion of longitudinal amplitudes}

In this appendix we give the multipole expansion of the longitudinal amplitudes $A_{5}^{\text {thr }}$ and $A_{6}^{\text {thr }}$ in the notation used to describe $A_{1}^{\text {thr }}$ in Eqs. (18) and (201).

$$
\begin{aligned}
& A_{5}^{\mathrm{thr}}=\frac{2 \pi(1+\mu)[2 \mu(2+\mu)+\rho]}{M_{N}^{2}\left[\mu^{2}(2+\mu)+\rho\right] \sqrt{(1+\mu)\left[(2+\mu)^{2}+\rho\right]}}\left\{\frac{2(1+\mu)}{M_{N}\left[\mu^{2}+\rho\right]} E_{0+}-\frac{4 \mu(1+\mu)^{2}}{M_{N}[\mu(2+\mu)-\rho]\left[\mu^{2}+\rho\right]} L_{0+}\right. \\
& -\frac{\mu(2-\mu) \sqrt{(2+\mu)^{2}+\rho}}{[2 \mu(2+\mu)+\rho] \sqrt{\mu^{2}+\rho}} \bar{P}_{2}-\frac{\mu(2+\mu)\left(4+8 \mu+\mu^{2}\right)+\left(4+2 \mu+\mu^{2}\right) \rho}{[2 \mu(2+\mu)+\rho] \sqrt{\left[(2+\mu)^{2}+\rho\right]\left[\mu^{2}+\rho\right]}} \bar{P}_{3} \\
& \left.+\frac{2 M_{N}(2+\mu)(2-\mu)}{2 \mu(2+\mu)+\rho} \bar{D}+\frac{8(1+\mu)^{2}(2+\mu)}{[\mu(2+\mu)-\rho] \sqrt{\left[(2+\mu)^{2}+\rho\right]\left[\mu^{2}+\rho\right]}} \bar{P}_{5}\right\} \\
& =\frac{2 \pi(1+\mu)}{M_{N}^{2}\left[\mu^{2}(2+\mu)+\rho\right] \sqrt{(1+\mu)\left[(2+\mu)^{2}+\rho\right]}}\left\{-\frac{2(1+\mu)[2 \mu(2+\mu)+\rho]}{M_{N}[\mu(2+\mu)-\rho]} E_{0+}\right. \\
& -\frac{\mu M_{N}[2 \mu(2+\mu)+\rho]\left[(2+\mu)^{2}+\rho\right]}{\mu(2+\mu)-\rho} \Delta_{0+}-\frac{\mu M_{N}(2-\mu)\left[(2+\mu)^{2}+\rho\right]}{2(1+\mu)} \mathcal{P}_{2} \\
& -\frac{M_{N}\left[\mu(2+\mu)\left(4+8 \mu+\mu^{2}\right)+\left(4+2 \mu+\mu^{2}\right) \rho\right]}{2(1+\mu)} \mathcal{P}_{3}+2 M_{N}(2+\mu)(2-\mu) \bar{D} \\
& \left.+\frac{4 M_{N}(1+\mu)(2+\mu)[2 \mu(2+\mu)+\rho]}{\mu(2+\mu)-\rho} \mathcal{P}_{5}\right\}, \\
& A_{6}^{\mathrm{thr}}=\frac{4 \pi}{M_{N}^{2}} \sqrt{\frac{1+\mu}{(2+\mu)^{2}+\rho}}\left\{\frac{2+\mu}{\mu^{2}+\rho} E_{0+}-\frac{2 \mu(1+\mu)(2+\mu)}{\left(\mu^{2}+\rho\right)[\mu(2+\mu)-\rho]} L_{0+}+\frac{\mu M_{N}}{2(1+\mu)} \sqrt{\frac{(2+\mu)^{2}+\rho}{\mu^{2}+\rho}} \bar{P}_{2}\right. \\
& \left.-\frac{\mu M_{N}}{2(1+\mu)} \sqrt{\frac{\mu^{2}+\rho}{(2+\mu)^{2}+\rho}} \bar{P}_{3}+\frac{\mu M_{N}^{2}}{1+\mu} \bar{D}-\frac{4 \mu M_{N}(1+\mu)(2+\mu)}{[\mu(2+\mu)-\rho] \sqrt{\left(\mu^{2}+\rho\right)\left[(2+\mu)^{2}+\rho\right]}} \bar{P}_{5}\right\} \\
& =4 \pi \sqrt{\frac{1+\mu}{(2+\mu)^{2}+\rho}}\left\{-\frac{2+\mu}{[\mu(2+\mu)-\rho] M_{N}^{2}} E_{0+}-\frac{\mu(2+\mu)\left[(2+\mu)^{2}+\rho\right]}{2(1+\mu)[\mu(2+\mu)-\rho]} \Delta_{0+}+\frac{\mu\left[(2+\mu)^{2}+\rho\right]}{4(1+\mu)^{2}} \mathcal{P}_{2}\right. \\
& \left.-\frac{\mu\left(\mu^{2}+\rho\right)}{4(1+\mu)^{2}} \mathcal{P}_{3}+\frac{\mu}{1+\mu} \bar{D}-\frac{2 \mu(2+\mu)}{\mu(2+\mu)-\rho} \mathcal{P}_{5}\right\} \text {. }
\end{aligned}
$$

\section{D - Pole contributions for S waves}

In the following we list the pole contributions and their expansions to $\mathcal{O}\left(q^{2}\right)$ for the threshold S-wave multipoles:

$$
\begin{aligned}
& E_{0+, \text { pole }}^{(+, 0) \mathrm{thr}}=-\frac{e g_{\pi N}}{16 \pi M_{N}} \frac{[\mu(2+\mu)-\rho]}{(2+\mu)(2+\mu+\rho)(1+\mu)^{3 / 2}}\left\{F_{1}^{V, S}\left(Q^{2}\right)+F_{2}^{V / S}\left(Q^{2}\right)\right\} \\
& =\frac{e g_{\pi N}}{32 \pi M_{N}}\left(-2 \mu+3 \mu^{2}+\rho\right)\left(1+\kappa_{V, S}\right)+\ldots, \\
& \underset{0+, \text { pole }}{(+, 0) \mathrm{thr}}=E_{0+, \text { pole }}^{(+, 0) \operatorname{thr}}-\frac{e g_{\pi N}}{64 \pi M_{N}}\left(\mu^{2}+\rho\right) \frac{[\mu(2+\mu)-\rho] \sqrt{(2+\mu)^{2}+\rho}}{(1+\mu)^{5 / 2}(2+\mu)(2+\mu+\rho)} F_{2}^{V, S}\left(Q^{2}\right) \\
& =\frac{e g_{\pi N}}{32 \pi M_{N}}\left(-2 \mu+3 \mu^{2}+\rho\right)\left(1+\kappa_{V, S}\right)+\ldots, \\
& E_{0+, \text { pole }}^{(-) \operatorname{thr}}=\frac{e g_{\pi N}}{16 \pi M_{N}} \frac{\left[(2+\mu)^{2}+\rho\right]^{3 / 2}}{(2+\mu)(2+\mu+\rho)(1+\mu)^{3 / 2}} \\
& \times\left\{F_{1}^{V}\left(Q^{2}\right)-\frac{(1+\mu) \rho}{(2+\mu)^{2}+\rho} F_{2}^{V}\left(Q^{2}\right)\right\} \\
& =\frac{e g_{\pi N}}{8 \pi M_{N}}\left\{1-\mu+\frac{9}{8} \mu^{2}-\frac{1}{4}\left(\kappa_{V}+\frac{1}{2}+\frac{2}{3} M_{N}^{2}\left\langle r^{2}\right\rangle_{1}^{V}\right) \rho+\ldots\right\} \text {, }
\end{aligned}
$$




$$
\begin{aligned}
L_{0+, \text { pole }}^{(-) \text {thr }}= & E_{0+, \text { pole }}^{(-) \text {thr }}-\frac{e g_{\pi N}}{16 \pi M_{N}}\left(\mu^{2}+\rho\right) \frac{\sqrt{(2+\mu)^{2}+\rho}}{(1+\mu)^{3 / 2}\left[\mu^{2}(2+\mu)+\rho\right]} \\
& \times\left\{F_{1}^{V}\left(Q^{2}\right)-\frac{\left[\mu^{2}(2+\mu)+\rho\right]\left[(2+\mu)^{2}+\rho\right]}{4(2+\mu)(2+\mu+\rho)(1+\mu)} F_{2}^{V}\left(Q^{2}\right)\right. \\
& \left.-\frac{\mu[\mu(2+\mu)-\rho]}{\rho(1+\mu)}\left(F_{\pi}^{V}\left(Q^{2}\right)-F_{1}^{V}\left(Q^{2}\right)\right)\right\} \\
= & E_{0+, \text { pole }}^{(-) \text {thr }}+\frac{e g_{\pi N}}{8 \pi M_{N}}\left(\mu^{2}+\rho\right)\left\{\frac{1}{4} \kappa_{V}-\frac{\sqrt{(2+\mu)^{2}+\rho}}{2(1+\mu)^{3 / 2}\left[\mu^{2}(2+\mu)+\rho\right]}\right. \\
& \left.+\frac{\rho}{6\left(2 \mu^{2}+\rho\right)} M_{N}^{2}\left\langle r^{2}\right\rangle_{1}^{V}+\frac{1}{6}\left[1-\frac{\rho}{2 \mu^{2}+\rho}\right] M_{N}^{2}\left(\left\langle r^{2}\right\rangle_{1}^{V}-\left\langle r^{2}\right\rangle_{\pi}^{V}\right)+\ldots\right\} .
\end{aligned}
$$

Strictly speaking the pion form factor can not be expanded in a power series, because it diverges in the chiral limit. However on the phenomenological level all the radii appearing in the above equations can be treated on the same footing.

\section{E - FFR multipoles}

Because the FFR amplitudes are independent of $t$, the associated current contributes only to the partial waves $0^{+}$and $1^{-}$corresponding to total angular momentum $\mathcal{J}=\frac{1}{2}$. The resulting multipole contributions are

$$
\begin{aligned}
& E_{0+, \mathrm{FFR}}^{(+, 0)}\left(W, Q^{2}\right)=\sqrt{\left(E_{i}+M_{N}\right)\left(E_{f}+M_{N}\right)} \frac{W-M_{N}}{8 \pi W} A_{1, \mathrm{FFR}}^{(+, 0)}\left(Q^{2}\right), \\
& \bar{M}_{1-, \mathrm{FFR}}^{(+, 0)}\left(W, Q^{2}\right)=-\sqrt{\frac{E_{i}-M_{N}}{E_{f}+M_{N}}} \frac{W+M_{N}}{8 \pi W} A_{1, \mathrm{FFR}}^{(+, 0)}\left(Q^{2}\right), \\
& L_{0+, \mathrm{FFR}}^{(+, 0)}\left(W, Q^{2}\right)=\sqrt{\left(E_{i}+M_{N}\right)\left(E_{f}+M_{N}\right)} \frac{k_{0}}{8 \pi W} A_{1, \mathrm{FFR}}^{(+, 0)}\left(Q^{2}\right), \\
& \bar{L}_{1-, \mathrm{FFR}}^{(+, 0)}\left(W, Q^{2}\right)=-\sqrt{\frac{E_{i}-M_{N}}{E_{f}+M_{N}}} \frac{k_{0}}{8 \pi W} A_{1, \mathrm{FFR}}^{(+, 0)}\left(Q^{2}\right), \\
& E_{0+, \mathrm{FFR}}^{(-)}\left(W, Q^{2}\right)=\sqrt{\left(E_{i}+M_{N}\right)\left(E_{f}+M_{N}\right)} \frac{Q^{2}}{8 \pi W} A_{6, \mathrm{FFR}}^{(-)}\left(Q^{2}\right), \\
& \bar{M}_{1-, \mathrm{FFR}}^{(-)}\left(W, Q^{2}\right)=\sqrt{\frac{E_{i}-M_{N}}{E_{f}+M_{N}}} \frac{Q^{2}}{8 \pi W} A_{6, \mathrm{FFR}}^{(-)}\left(Q^{2}\right), \\
& L_{0+, \mathrm{FFR}}^{(-)}\left(W, Q^{2}\right)=-\sqrt{\left(E_{i}+M_{N}\right)\left(E_{f}+M_{N}\right)} \frac{\left(W-M_{N}\right) k_{0}}{8 \pi W} A_{6, \mathrm{FFR}}^{(-)}\left(Q^{2}\right), \\
& \bar{L}_{1-, \mathrm{FFR}}^{(-)}\left(W, Q^{2}\right)=-\sqrt{\frac{E_{i}-M_{N}}{E_{f}+M_{N}}} \frac{\left(W+M_{N}\right) k_{0}}{8 \pi W} A_{6, \mathrm{FFR}}^{(-)}\left(Q^{2}\right) .
\end{aligned}
$$

where $A_{1, \mathrm{FFR}}^{(+, 0)}$ and $A_{6, \mathrm{FFR}}^{(-)}$are given in Eqs. (24) and (25) , respectively. Note that the longitudinal multipoles vanish at $k_{0}=0$ or $Q^{2}=W^{2}-M_{N}^{2}$, and that the P-waves vanish at pseudo-threshold, $E_{i}=M_{N}$ or $Q^{2}=-\left(W-M_{N}\right)^{2}$.

\section{F - Expansion of CGLN amplitudes in terms of invariant amplitudes}

The CGLN amplitudes are obtained from the invariant amplitudes by the following equations [3,5]:

$$
\begin{aligned}
& \mathcal{F}_{1}=\frac{W-M_{N}}{8 \pi W} \sqrt{\left(E_{i}+M_{N}\right)\left(E_{f}+M_{N}\right)} \\
& \quad \times\left\{A_{1}+\left(W-M_{N}\right) A_{4}-\frac{2 M_{N} \nu_{B}}{W-M_{N}}\left(A_{3}-A_{4}\right)+\frac{Q^{2}}{W-M_{N}} A_{6}\right\}, \\
& \mathcal{F}_{2}=\frac{W+M_{N}}{8 \pi W} q \sqrt{\frac{E_{i}-M_{N}}{E_{f}+M_{N}}}
\end{aligned}
$$




$$
\begin{aligned}
& \times\left\{-A_{1}+\left(W+M_{N}\right) A_{4}-\frac{2 M_{N} \nu_{B}}{W+M_{N}}\left(A_{3}-A_{4}\right)+\frac{Q^{2}}{W+M_{N}} A_{6}\right\}, \\
& \mathcal{F}_{3}=\frac{W+M_{N}}{8 \pi W} q \sqrt{\left(E_{i}-M_{N}\right)\left(E_{f}+M_{N}\right)} \\
& \times\left\{\frac{2 W^{2}-2 M_{N}^{2}+Q^{2}}{2\left(W+M_{N}\right)} A_{2}+A_{3}-A_{4}-\frac{Q^{2}}{W+M_{N}} A_{5}\right\}, \\
& \mathcal{F}_{4}=\frac{W-M_{N}}{8 \pi W} q^{2} \sqrt{\frac{E_{i}+M_{N}}{E_{f}+M_{N}}} \\
& \times\left\{-\frac{2 W^{2}-2 M_{N}^{2}+Q^{2}}{2\left(W-M_{N}\right)} A_{2}+A_{3}-A_{4}+\frac{Q^{2}}{W-M_{N}} A_{5}\right\}, \\
& \mathcal{F}_{5}=\frac{k_{0}}{8 \pi W} \sqrt{\frac{E_{f}+M_{N}}{E_{i}+M_{N}}} \\
& \times\left\{\left(E_{i}+M_{N}\right) A_{1}+\left[4 M_{N} \nu_{B}\left(W-\frac{3}{4} k_{0}\right)-k^{2} W+q_{0}\left(W^{2}-M_{N}^{2}+\frac{1}{2} Q^{2}\right)\right] A_{2}+\right. \\
& {\left[q_{0}\left(W+M_{N}\right)+2 M_{N} \nu_{B}\right] A_{3}+\left[\left(E_{i}+M_{N}\right)\left(W-M_{N}\right)-q_{0}\left(W+M_{N}\right)-2 M_{N} \nu_{B}\right] A_{4}+} \\
& \left.\left(2 M_{N} \nu_{B} k_{0}-q_{0} Q^{2}\right) A_{5}-\left(E_{i}+M_{N}\right)\left(W-M_{N}\right) A_{6}\right\}, \\
& \mathcal{F}_{6}=\frac{k_{0} q}{8 \pi W \sqrt{\left(E_{f}+M_{N}\right)\left(E_{i}-M_{N}\right)}} \\
& \times\left\{-\left(E_{i}-M_{N}\right) A_{1}+\left[k^{2} W-4 M_{N} \nu_{B}\right)\left(W-\frac{3}{4} k_{0}\right)-q_{0}\left(W^{2}-M_{N}^{2}+\frac{1}{2} Q^{2}\right)\right] A_{2}+ \\
& {\left[q_{0}\left(W-M_{N}\right)+2 M_{N} \nu_{B}\right] A_{3}+\left[\left(E_{i}-M_{N}\right)\left(W+M_{N}\right)-q_{0}\left(W-M_{N}\right)-2 M_{N} \nu_{B}\right] A_{4}+} \\
& \left.\left(q_{0} Q^{2}-2 M_{N} \nu_{B} k_{0}\right) A_{5}-\left(E_{i}-M_{N}\right)\left(W+M_{N}\right) A_{6}\right\},
\end{aligned}
$$

with $\nu_{B}=\left(t-M_{\pi}^{2}+Q^{2}\right) /\left(4 M_{N}\right)$. 